\title{
Long non-coding RNA Gm15441 attenuates hepatic inflammasome activation in response to metabolic stress
}

\section{Authors}

Chad N. Brocker ${ }^{1 \#}$, Donghwan Kim ${ }^{1 \#}$, Tisha Melia ${ }^{2}$, Kritika Karri² ${ }^{2}$ Thomas J. Velenosi ${ }^{1}$, Shogo

Takahashi $^{1}$, Jessica A. Bonzo ${ }^{1}$, David J. Waxman ${ }^{2}$, and Frank J. Gonzalez ${ }^{1 *}$

${ }^{1}$ Laboratory of Metabolism, Center for Cancer Research, National Cancer Institute, National Institutes of Health, Bethesda, Maryland 20892

${ }^{2}$ Department of Biology and Bioinformatics Program, Boston University, Boston, Massachusetts 02215

Running title: PPARA reduces inflammation through induction of lncRNA Gm15441

\#These authors contributed equally

*Correspondence: gonzalef@mail.nih.gov 


\section{Summary}

Fasting paradigms elicit a wide-range of health benefits including suppressing inflammation. Exploring the molecular mechanisms that prevent inflammation during caloric restriction may yield promising new therapeutic targets. During fasting, activation of the nuclear receptor peroxisome proliferator-activated receptor alpha (PPARA) promotes the utilization of lipids as an energy source. Herein, we show that ligand activation of PPARA directly upregulates the long non-coding RNA gene Gm15441 through binding sites within its promoter. Gm15441 expression suppresses its antisense transcript, encoding thioredoxin interacting protein (TXNIP). This, in turn, decreases TXNIP-stimulated NLRP3 inflammasome activation, caspase-1 (CASP1) cleavage, and proinflammatory interleukin 1 beta (IL1B) maturation. Gm15441-null mice were developed and shown to be more susceptible to NLRP3 inflammasome activation and to exhibit elevated CASP1 and IL1B cleavage in response to metabolic and inflammatory stimuli. These findings provide evidence for a novel mechanism by which PPARA attenuates hepatic inflammasome activation in response to metabolic stress through lncRNA Gm15441 induction.

\section{Keywords:}

caloric restriction; fasting; inflammasome; inflammation; lncRNA; NLRP3; PPAR; PPARA; TXNIP; WY-14643

\section{Introduction}

There is growing support for a strong link between metabolism and inflammation (Andrejeva and Rathmell, 2017; Bettencourt and Powell, 2017). Various fasting regimes are known to provide many health benefits including anti-inflammatory effects (Corrales et al., 2019). Peroxisome proliferator-activated receptor alpha (PPARA) is a ligand-activated nuclear receptor and transcription factor that is a key regulator of the fasting response. PPARA facilitates metabolic remodeling that promotes lipid oxidation, and its dysregulation contributes to metabolic disorders and liver disease (Brocker et al., 2018). Further, synthetic PPARA agonists act as potent antiinflammatory agents (Yang et al., 2017; Zheng et al., 2017). However, the regulatory mechanisms underlying how PPARA prevents inflammation are not well understood. 
Long non-coding RNAs (lncRNAs) act as regulators of gene expression and play important regulatory roles in many metabolic processes. For example, lncRNA Blnc1 works in concert with another important hepatic nuclear receptor, liver X receptor (LXR), to activate the lipogenic gene program (Zhao et al., 2018). Down-regulation of lncRNA lncOb reduces leptin, leading to a leptin responsive form of obesity (Dallner et al., 2019). Other studies found that lncRNAs are regulated during adipocyte differentiation (Yuan et al., 2019a; Yuan et al., 2019b), are expressed in liver in a sex-dependent manner when regulated by growth hormone (Melia et al., 2016; Melia and Waxman, 2019), and can be strongly induced by xenobiotic exposure (Lodato et al., 2017). A compartment-specific transcriptional profiling approach revealed that lncRNA PAXIP1-AS1 regulates pulmonary arterial hypertension by modulating smooth muscle cell function (Jandl et al., 2019). Further, lncRNA HOTAIR influences glucose metabolism by upregulation of GLUT1 in hepatocellular carcinoma cells (Wei et al., 2017). It is therefore reasonable to consider that lncRNAs may play important roles in the metabolic remodeling and anti-inflammatory actions that occur after PPARA activation.

In the present study, the mechanisms by which PPARA prevents inflammation during periods of metabolic stress were investigated. RNA-seq was carried out on livers from mice treated with the PPARA agonist WY-14643, and large numbers of differentially expressed protein coding genes and lncRNA genes were identified. These studies led to discovery of a regulatory axis between thioredoxin-interacting protein (TXNIP) and an antisense lncRNA, Gm 15441. TXNIP acts as a critical relay linking oxidative and endoplasmic reticulum (ER) stress to inflammation through NLRP3 inflammasome activation (Anthony and Wek, 2012; Oslowski et al., 2012). Studies have also shown that NLRP3 inflammasome activity is attenuated by mono- and polyunsaturated fatty acids, which are endogenous PPARA agonists (Forman et al., 1997; Kliewer et al., 1997; Mochizuki et al., 2006; Ralston et al., 2017; Shen et al., 2017). Considering these studies, a regulatory mechanism was proposed whereby fatty acids mobilized from adipose tissue during fasting activate PPARA, which in turn suppresses the NLRP3 inflammasome by strong induction of the TXNIP-suppressing lncRNA gene Gm15441, which is antisense to Txnip. ChIPseq datasets were analyzed for PPARA binding sites within the Gm15441 promoter and Gm15441 regulatory elements were confirmed using reporter gene assays and PPARA ChIP studies. Gm15441 transgene expression downregulated Txnip, demonstrating lncRNA-mediated gene suppression in vitro. CRIPSR/Cas9-mediated gene editing was employed to develop a Gm15441 knockout $\left(G m 15441^{\mathrm{LSL}}\right)$ mouse model. Basal TXNIP levels in Gm15441 ${ }^{\mathrm{LSL}}$ mice were significantly elevated over wild-type mice levels, supporting Gm15441 as a negative regulator of 
Txnip expression in vivo. Gm15441 $1^{\mathrm{LSL}}$ mice were treated with a PPARA agonist or fasted to assess how loss of Gm15441 impacts hepatic inflammasome activation in response to both pharmacological and physiologically-induced metabolic stress. TXNIP protein, caspase-1 (CASP1) levels, and interleukin 1 beta (IL1B) cleavage were elevated in Gm $15441^{\text {LSL }}$ mice and were further increased by PPARA activation, indicating that this lncRNA plays a major role in attenuating inflammasome activation. Thus, hepatic PPARA directly regulates the lncRNA Gm15441, which in turn suppresses Txnip expression, which attenuates NLRP3 inflammasome activation during periods of metabolic stress. These studies revealed a novel regulatory mechanism supporting the beneficial effects of fasting, namely, reduced inflammation.

\section{Results}

\section{LncRNA regulation by PPARA is highly tissue-specific}

To assess the regulation of lncRNAs by PPARA, RNA-seq was performed using total liver RNA isolated from Ppara $^{+/+}$mice, both with and without dietary exposure to the PPARA agonist WY-14643. A lncRNA discovery pipeline was implemented that identified 15,558 liver-expressed lncRNA genes. Of these, 13,343 were intergenic, 1,966 were antisense, and 249 were intragenic lncRNAs. $44 \%$ of the 15,558 liver-expressed lncRNAs are considered novel (Melia and Waxman, 2019). Differential gene expression analysis revealed that a total of 1,735 RefSeq genes and 442 liver-expressed lncRNA genes responded to treatment with WY-14643 at an expression foldchange $>2$ at FDR $<0.05$, with 968 RefSeq genes and 245 lncRNA genes upregulated, and 767 RefSeq genes and 197 lncRNA genes downregulated following WY-14643 treatment (Table S1A and Table S1B). Only 17 RefSeq genes (1.0\%) and 6 lncRNAs (1.3\%) responded to WY-14643 in the same manner in PPARA-knockout mice. Thus, $\sim 99 \%$ of these transcriptomic responses are PPARA-dependent (Figure 1A).

Comparison of the PPARA-responsive lncRNAs to lncRNAs responsive to agonist ligands of two other nuclear receptors in mouse liver, namely CAR and PXR (Lodato et al., 2017), identified 8 lncRNAs that are induced by all three nuclear receptors and $11 \operatorname{lncRNAs}$ repressed by all three receptors. Forty five other lncRNAs were induced, or were repressed, in common by PPARA and CAR, and 30 other lncRNAs were either induced or repressed in common by PPARA and PXR (Table S1C). Overall, 94 (21\%) of the 442 PPARA-responsive liver-expressed lncRNAs showed common responses to CAR and/or PXR activation. Furthermore, 59 other PPARAresponsive lncRNAs showed opposite patterns of response to PPARA as compared to CAR or 
PXR (Table S1C). These findings are consistent with the partial functional overlap between these three xenobiotic nuclear receptors and their gene targets (Cui and Klaassen, 2016; Gonzalez et al., 2015; Wada et al., 2009).

Pathway analysis revealed that the WY-14643 upregulated genes are most highly enriched for the following biological processes: lipid metabolism, peroxisome, DNA replication, DNA repair, cell cycle and fatty acid oxidation (Table S1D). The downregulated genes are most highly enriched for: secreted factors, monooxygenase, immunity, serine protease inhibitor, metabolism of xenobiotics, and response to virus (Table S1E). PPARA-dependent lncRNAs identified by RNA-seq were selected and mRNA levels monitored over a 24 hour period after WY-14643 treatment, revealing that lncRNA expression profiles paralleled those of known protein-coding PPARA target genes (Figure S1A-D). As lncRNA expression is often highly tissue specific (Iwakiri et al., 2017; Melia et al., 2016; Perron et al., 2017), the relative basal levels were measured for PPARA-responsive lncRNAs expressed in oxidative tissues that utilize fatty acids as an energy source and express PPARA at the highest levels. Ppara mRNA and its classic target gene Cyp $4 a 14$ served as controls. Basal levels of Cyp4a14 mRNA and intergenic lncRNAs ncRNA_chr19_15132 and $n c R N A \_c h r 4 \_3651$ were highest in liver, while $n c R N A \_c h r 9 \_8249$, ncRNA_chr9_8081, and 1300002E11Rik were most highly expressed in muscle. One of the PPARA-dependent lncRNAs, Gm15441, was also most highly expressed in brown adipose tissue (BAT) (Figure 1B).

To assess the impact of PPARA activation on lncRNA expression and tissue specificity, Ppara wild-type mice $\left(\right.$ Ppara $\left.^{+/+}\right)$and Ppara-null mice $\left(\right.$Ppara $\left.^{-/-}\right)$were treated with WY-14643 for 48 hours, and four tissues (liver, BAT, heart, muscle) then harvested for analysis. Cyp 4 al 4 mRNA was markedly induced by WY-14643 in all four tissues and in a Ppara-dependent manner, as expected (Figure 1C). All six lncRNAs were induced in liver, including Gm 15441, which exhibited $>2000$-fold increase in wild-type livers, $>10$-fold higher than the other lncRNAs. In contrast to the induction of Cyp4a14 mRNA, lncRNA induction by WY-14643 was liver-specific, with no significant induction of the five other lncRNAs seen in BAT, heart, or muscle (Figure 1C). Further, none of these lncRNAs was induced in Ppara $^{-/-}$mice in any tissue examined. Thus, PPARA-mediated induction of these lncRNAs is highly liver-specific, with Gm15441 showing the most robust induction response.

\section{LncRNA Gm15441 is antisense to Txnip and exhibits inverse regulation in response to PPARA activation}


LncRNA Gm 15441 overlaps the coding region of Txnip, which is located on the opposing strand and also responds to WY-14643 treatment, albeit with different kinetics than Gm15441. Analysis of the mapped sequence reads obtained by stranded RNA sequencing revealed a pronounced increase in expression of Gm15441 (Figure 2A), whereas, the Txnip transcript on the opposite strand was suppressed by WY-14643 at the time point analyzed (Figure 2B). qRT-PCR analysis confirmed the very large, highly significant increase in Gm15441 expression and its complete inhibition in Ppara $^{-/-}$liver (Figure 2C). Further, Txnip RNA was significantly suppressed by WY-14643 in wild-type mice (Figure 2D). Additionally, Txnip expression was attenuated in untreated Ppara $^{-/-}$compared to Ppara $^{+/+}$mouse liver, indicating a role for PPARA in maintaining basal expression of Txnip (Figure 2D). Given that Txnip expression can be upregulated in human neuroblastoma cells by the PPARA activator fenofibrate, which suppresses proliferation and migration (Su et al., 2015), the effects of a single dose of WY-14643 on Gm15441 and Txnip mRNA were examined over a 24 hour period. Txnip mRNA was induced rapidly, with maximum expression seen at 1.5 hours, followed by a marked decrease coincident with the increased expression of Gm15441 (Figure 2E). This time course is markedly different from that of 14 other WY-14643-inducible protein coding and lncRNA genes examined, where peak induction occurred 6 to 12 hours after WY-14643 treatment (Figure S1), as was also found for Gm15441. The unusual kinetics seen with Txnip - early induction followed by repression coinciding with the activation of Gm15441 - indicate that Txnip is a PPARA target gene whose expression in liver is inversely regulated with that of Gm15441 after PPARA activation.

\section{PPARA directly regulates IncRNA $G m 15441$ by binding to its promotor.}

To identify PPARA binding sites at the Gm15441/Txnip locus, PPARA ChIP-seq datasets (GSE61817) from PPARA agonist-treated mice (Lee et al., 2014a) were analyzed (Figure 3A). Three major ChIP-seq peaks indicating PPARA binding were seen upstream of Gm15441, and one major peak was seen in the promoter region of Txnip. Examination of genomic sequences upstream of Gm15441 using Genomatix MatInspector (Genomatix, Munchen, Germany) identified seven peroxisome proliferator response elements (PPREs) within six genomic regions, designated A to F, within 10 kilobase (kb) upstream of the Gm15441 transcriptional start site (TSS) (Figure 3B). These six Gm15441 upstream sequences were synthesized and cloned into the pGL4.11 reporter, and luciferase assays were performed to assay the functionality of PPARA binding to the Gm15441 promoter. A PPRE-luciferase construct containing an Acoxl PPRE repeat was used as a positive 
control, and an empty pGL4.11 plasmid was used as a negative control. Luciferase activity was significantly elevated in primary mouse hepatocytes transfected with five of the six pGL4.11 constructs, consistent with direct regulation by PPARA at multiple loci (Figure 3C). PPARA binding was also assessed by ChIP assays using a polyclonal PPARA antibody and chromatin isolated from livers of wild-type and $\mathrm{Ppara}^{-/-}$mice fed either control diet or a diet-containing WY14643. Enrichment of PPARA binding to PPREs of known PPARA target genes, namely Acot1 and Acoxt1, was determined by comparing binding to liver chromatin from wild-type mice fed control diet vs. WY-14643-containing diet (Figure 3D). Ppara $^{-/-}$livers were used as a negative control to identify non-specific binding. Fscn2 primers were used as a non-target gene promoter and negative control. Enrichment of PPARA binding was found using primer sets covering Gm 15441 regions B, C, D, and F, while no binding was seen with Gm15441 region E (Figure 3D), which was transcriptionally inactive (Figure 3C). Enrichment was strongest with region $\mathrm{C}$, in agreement with the luciferase reporter gene data. Enrichment at sites C and D was significantly increased by WY-14643 treatment, indicating increased PPARA recruitment at these sites. No PPARA binding was seen with chromatin from Ppara $^{-/-}$mice on the control diet. Together, these data indicate that PPARA regulates Gm15441 transcription by direct binding of PPARA to multiple PPRE sites within $10 \mathrm{~kb}$ of the Gm15441 TSS.

\section{Generation of a strand-specific Gm15441 knockout mouse.}

A gene targeting strategy was developed to generate a Gm15441 knockout mice without impacting Txnip expression on the opposing strand. To accomplish this goal, CRISPR/Cas9 was used to insert a lox-STOP-lox (LSL) cassette downstream of Gm15441 exon 1 (Figure 4A and B). The LSL cassette prevents transcription of Gm15441 in a Gm15441-floxed mouse but has no effect on transcription of Txnip, which is $>2 \mathrm{~kb}$ downstream of the last exon of Txnip. Crossing with a transgenic Cre mouse deletes the LSL cassette and restores Gm15441 expression (Figure 4C). All mouse lines were on a pure C57BL/6J background. The genotyping scheme generates an approximately 1200 bp band in $G m 15441$ wild-type mice $\left(G m 15441^{+/+}\right)$and a 1700 bp band in Gm $15441^{\mathrm{LSL}}$ mice (Figure 4D). Basal expression of Gm15441 was significantly lower in livers of Gm $15441^{\mathrm{LSL}}$ when compared to $G m 15441^{+/+}$mice (Figure 4E). Expression in heterozygous $G m 15441^{\mathrm{HET}}$ mice was comparable to $G m 15441^{\mathrm{LSL}}$. To verify the subcellular localization and loss of expression of Gm15441, fluorescence in situ hybridization (FISH) staining was performed on livers from $G m 15441^{+/+}$and $G m 15441^{\text {LSL }}$ mice fed control diet or treated with WY-14643. DAPI 
staining was used as a counter stain to detect nuclei in liver sections. A pronounced increase in Gm15441 fluorescence was detected in the cytoplasm and nuclei of hepatocytes in livers from WY-14643-treated $G m 15441^{+/+}$mice. The Gm15441 RNA signal was absent in livers from vehicle-treated $G m 15441^{+/+}$and $G m 15441^{\text {LSL }}$ mice, and in livers from WY-14643-treated Gm $15441^{\mathrm{LSL}}$ mice (Figure 4F). These data validate $\mathrm{Gm} 15441^{\mathrm{LSL}}$ mice as an effective knockout mouse model and reveal that hepatic Gm15441 expression is both nuclear and cytosolic evoking several possible mechanisms by which it regulates Txnip.

\section{Loss of Gm15441 potentiates inflammasome activation by WY-14643-induced metabolic stimulation.}

PPARA plays a role in attenuating inflammation in many tissues and disease models (Kono et al., 2009; Schaefer et al., 2008; Yoo et al., 2013; Yoo et al., 2011). In the liver, PPARA ameliorates inflammation by reducing ER stress in hepatocytes (Zhang et al., 2016). TXNIP is known to facilitate ER stress-induced NLRP3 inflammasome activation (Anthony and Wek, 2012; Lee et al., 2014b; Lerner et al., 2012; Oslowski et al., 2012). By regulating TXNIP levels, Gm15441 could serve as a link between PPARA and the NLRP3 inflammasome. To ascertain whether lncRNA Gm15441 attenuates inflammasome activation by regulating TXNIP levels, mice were treated with WY-14643 for 48 hours, and weight loss, liver index, ALT, AST, and serum glucose levels were measured. There was no discernable difference in weight loss between Gm $15441^{+/+}$and Gm $15441^{\text {LSL }}$ mice on a WY-14643 diet (Figure 5A). Serum ALT and AST levels were unchanged by genotype or following WY-14643 treatment. Serum glucose levels were significantly decreased by WY-14643 in both $G m 15441^{+/+}$and $G m 15441^{\mathrm{LSL}}$ mice, but the decrease was significantly greater in $G m 15441^{\mathrm{LSL}}$ mice (Figure 5A). Furthermore, upon gross liver examination, pronounced hepatomegaly was induced by WY-14643 treatment in both $G m 15441^{+/+}$ and $G m 15441^{\text {LSL }}$ mice (Figure 5B). Pronounced swelling of hepatocytes was noted in livers of WY-14643-treated $G m 15441^{+/+}$and $G m 15441^{\text {LSL }}$ mice (Figure 5B). Expression of the PPARA target genes acyl-CoA dehydrogenase medium chain (Acadm) and Cyp4a14 was significantly increased by WY-14643 in both $G m 15441^{+/+}$and $G m 15441^{\text {LSL }}$ mice. Gm15441 was strongly upregulated in livers of wild-type mice but was not detected in Gm15441 ${ }^{\mathrm{LSL}}$ mice. Txnip mRNA was significantly decreased by WY-14643 in livers of wild-type animals, consistent with Figure 2, but was significantly induced in $G m 15441^{\text {LSL }}$ livers (Figure 5C). Basal expression of the inflammasome-related proteins TXNIP, cleaved caspase 1 (CASP1), and cleaved interleukin $1 \beta$ 
(IL1B) was elevated in livers of $G m 15441^{\mathrm{LSL}}$ mice compared to wild-type mice, and significant further increases were seen following WY-14643 treatment (Figure 5D, 5E). Overall, levels of all three proteins were much higher in livers of WY-14643-treated $G m 15441^{\mathrm{LSL}}$ mice than wild-type mice (Figure 5E). Thus, ablation of Gm15441 increases the expression of TXNIP and two other proteins associated with NLRP3 inflammasome activation, namely, cleaved CASP1 and IL1B.

\section{Loss of Gm15441 potentiates inflammasome activation during physiological response to acute fasting.}

During fasting, hepatic PPARA facilitates the metabolic remodeling that promotes use of lipids as an alternate energy source (Brocker et al., 2018). Fasting is also associated with many anti-inflammatory effects (Corrales et al., 2019). To determine whether Gm15441 regulates inflammasome activation during physiological fasting, $G m 15441^{+/+}$and $G m 15441^{\mathrm{LSL}}$ mice were fasted for 24 hours. Serum ALT and AST were not changed between $\mathrm{Gm}_{15441^{+/+}}$and Gm 15441 ${ }^{\text {LSL }}$ mice, both with and without WY-14643 treatment (Figure 6A). Fasting decreased serum glucose levels in both $G m 15441^{+/+}$and $G m 15441^{\mathrm{LSL}}$ mice, but the decrease was greater in Gm $15441^{\mathrm{LSL}}$ mice (Figure 6A). Expression of Acadm, Cyp4a14, and Txnip mRNAs was significantly increased by fasting in both $G m 15441^{+/+}$and $G m 15441^{\mathrm{LSL}}$ mice (Figure S2A). Gm15441 was significantly induced by fasting, although to a much lower degree than with WY14643 treatment (Figure S2A). Expression of Txnip mRNA was increased to the same level in livers of fasted $G m 15441^{\mathrm{LSL}}$ mice as in fasted $G m 15441^{\mathrm{LSL}}$ mice (Figure S2A). Fasting also increased TXNIP, CASP1, and IL1B protein levels in $G m 15441^{\mathrm{LSL}}$ mice, in contrast to either a modest increase (TXNIP) or no increase (CASP1, IL1B) in Gm $15441^{+/+}$mice (Figure S2B, Figure S2C). Fasting markedly promotes lipid accumulation in mouse liver (Brocker et al., 2018). Lipid droplet accumulation was also observed and more widespread in fasting $G m 15441^{\mathrm{LSL}}$ mice than fasting $G m 15441^{+/+}$mice (Figure S2D). ORO staining was performed to validate the lipid accumulation response to fasting in $G m 15441^{\mathrm{LSL}}$ mice. A greater amount of lipid accumulation was observed in fasting Gm15441 $1^{\mathrm{LSL}}$ liver than in fasting $G m 15441^{+/+}$mice liver (Figure 6B). Serum and liver triglyceride (TG) levels and liver (but not serum) total cholesterol (CHOL) levels were significantly elevated in fasting $G m 15441^{\text {LSL }}$ mouse livers compared to fasting $G m 15441^{+/+}$ mouse livers (Figure 6C). These findings were supported by RNA-sequencing analysis, which revealed that liver damage and lipid accumulation-related genes were impacted by Gm15441 deficiency, albeit in a complex manner (Table S2). Furthermore, analysis of the impact of fasting 
on mRNAs for apolipoprotein A4 (Apoa4), betaine-homocysteine methyltransferase (Bhmt), lipocalin 2 (Lcn2), orosomucoid 2 (Orm2), serum amyloid A1 (Saal), and serum amyloid A2 (Saa2) - biomarker genes for liver damage, inflammation, and/or fatty accumulation - showed these genes were significantly elevated in fasting $G m 15441^{\mathrm{LSL}}$ compared to $\mathrm{Gm} 15441^{+/+}$mouse livers (Figure 6D). Fasting induced lower but still significant increases in several of these mRNAs in $G m 15441^{+/+}$livers, which could reflect the fasting-induced increase in Txnip expression (Figure S2). Thus, Gm15441 negatively regulates the NLRP3 inflammasome pathway and lipid accumulation, potentially by preventing Txnip expression, in response to fasting.

\section{Translational regulation of TXNIP by Gm15441 is partially dependent on 5' UTR sequences}

Translational regulation of TXNIP can occur via an internal ribosome entry site (IRES) sequences found in the 5'UTR (Lampe et al., 2018). IRES sequences are commonly associated with cell survival- and stress response-related genes and utilized when cap-dependent translation is inhibited during nutrient deprivation (Wu et al., 2014). The 5'UTR of Txnip contains multiple binding sites for polypyrimidine tract-binding protein (PTB), which regulates IRES-mediated translation (Lampe et al., 2018). As Gm15441 transcription generates an RNA antisense to the 5'UTR of Txnip, Gm15441 RNA may block PTB binding to the Txnip 5'UTR and thereby inhibit Txnip translation. To investigate this regulatory mechanism, the translational inhibitory potential of the Txnip 5'UTR was examined when linked to the coding sequence for GFP (Figure 7A). The non-5'UTR-GFP and the 5'UTR-GFP expression vectors were each transfected into Hepa-1 cells together with a $G m 15441$ expression vector or an empty expression plasmid. After 48 hours, strong Gm15441 expression was seen, but had no effect on the RNA levels of GFP or UTR-GFP (Figure 7B). In contrast, GFP protein translated from the Txnip 5'UTR-GFP template was significantly reduced in cells co-transfected with Gm15441 expression plasmid (Figure 7C). This 5'UTRdependent decrease in GFP protein was most striking when examined by fluorescent microscopy (Figure 7D and E). These findings suggest Gm15441 may regulate TXNIP translation through IRES sites found within its 5'UTR. To examine whether lncRNA Gm 15441 impacts expression of genes that flank Gm15441 on mouse chromosome 3, namely, Hfe2 (Hjv), Pol3gl and Ankrd34a, Hepa-1 cells and NIH3T3 mouse embryonic fibroblast cells were transfected with Gm15441 expression vector, or empty plasmid. The Gm15441 transgene was expressed and significantly downregulated Txnip without impacting the expression of $\mathrm{Hfe} 2$, Pol3gl, or Ankrd34a (Figure 7F). 
Together, these data support a regulatory mechanism whereby PPARA induces Gm15441, which in turn attenuates inflammation through TXNIP and the NLRP3 inflammasome pathway (Figure 7G).

\section{Discussion}

The physiological alterations that accompany fasting impart several health benefits, including anti-inflammatory effects (Goodpaster and Sparks, 2017; Montagner et al., 2016). As such, the underlying molecular pathways modulated by caloric restriction may present promising new therapeutic targets. PPARA activation during fasting is a key regulatory event of lipid and glucose metabolism. A growing body of evidence indicates that PPARA activation also potently suppresses inflammation in several disease models and tissues (Abcouwer, 2013; Krysiak et al., 2011; Lee et al., 2007; Tomizawa et al., 2011; Zhao et al., 2017). However, the mechanism by which PPARA modulates metabolic stress-induced inflammation is not known. PPARA is activated by endogenous fatty acid metabolite ligands in response to fasting and promotes the uptake, utilization, and catabolism of fatty acids by regulating a wide range of genes that reprogram metabolic pathways to facilitate the use of lipids as an energy source. The direct regulation of protein-coding genes by PPARA is well characterized, but it was not known whether lncRNAs which may influence gene expression through a variety of mechanisms (Li et al., 2017b; Militello et al., 2018; Zhao et al., 2016) - also serve as direct targets contributing to physiological changes induced by PPARA activation. This possibility was suggested by the finding that several hundred liver-expressed lncRNAs are dysregulated in livers exposed to xenobiotic agonist ligands of the nuclear receptors CAR and PXR (Lodato et al., 2017), which contribute to the regulation of lipid metabolism and whose gene targets overlap with those of PPARA (Cui and Klaassen, 2016; Wada et al., 2009). The present study identified several hundred PPARA-responsive liver-expressed lncRNA genes, including anti-sense lncRNAs, which often contribute to the regulation of genes on the opposing strand (Guil and Esteller, 2012; Qu et al., 2019; Zhang et al., 2017a). Further, a novel PPARA-dependent regulatory axis involving one such anti-sense lncRNA, Gm15441, was characterized. Gm15441 was shown to be transcribed in a liver-specific, PPARA-dependent manner to yield an anti-sense lncRNA that protects against metabolic stress by suppressing PPARA induction of the opposing, sense transcript, TXNIP, and thereby suppresses TXNIPmediated NLRP3 inflammasome activation. 
To ascertain whether PPARA regulates lncRNAs, RNA-seq was performed on livers from wild-type and Ppara $^{-/-}$mice treated with the specific PPARA agonist WY-14643. More than 400 liver-expressed lncRNAs, including many novel non-coding RNA transcripts, were significantly upregulated or downregulated in mouse liver 48 hours after WY-14643 treatment. Only six of these lncRNAs were similarly responsive to WY-14643 in Ppara $^{-/-}$mice, thus establishing the striking PPARA dependence of these non-coding RNA transcriptomic responses. PPARAdependent, liver-specific expression was verified for six non-coding RNAs, and detailed functional studies were carried out on one such gene, Gm15441, which showed an unusually strong induction in WY-14643-treated liver. Importantly, the genomic orientation of Gm15441 is antisense to that of Txnip, which codes for a ubiquitously-expressed protein that facilitates cellular responses to oxidative stress and inflammation (Watanabe et al., 2010). TXNIP was originally identified as a negative regulator of thioredoxin (Muoio, 2007), and subsequent studies found that TXNIP contributes to a wide array of processes in several tissues. Notably, TXNIP causes an increase in intracellular reactive oxygen species generation (Hong et al., 2016) that may inhibit hepatocellular carcinoma cell proliferation ( $\mathrm{Li}$ et al., 2017a). TXNIP is key regulator of NLRP3 inflammasome activation, which plays an important role in liver fibrosis and hepatocellular carcinoma (Ringelhan et al., 2018; Wree et al., 2017), and its activation is associated with the NLRP3 inflammasome pathway in human diseases (Bai et al., 2019; Kim et al., 2019; Li et al., 2019). TXNIP has also emerged as an important glucose sensor that regulates glucose uptake in response to insulin (Waldhart et al., 2017) and plays an important role in metabolic stress (Mandala et al., 2016; Wu et al., 2013). Another study found that PPARA activation by fenofibrate downregulated Txnip mRNA and TXNIP protein in endothelial cells (Deng et al., 2017), suggesting the Gm15441-Txnip regulatory axis described here for liver may also function in extrahepatic tissues. An earlier study found that Ppara mRNA and PPARA target gene mRNAs were elevated in Txnip-null mice, and that Txnip expression attenuated PPRE-luciferase response when co-transfected with PPARA/RXR and treated with WY-14643 (Oka et al., 2009), suggesting a Txnip-Ppara feedback regulatory loop that may also involve Gm15441.

LncRNAs regulate diverse cellular processes, including metabolism-related genes expressed on the opposing strand (Marin-Bejar et al., 2017; Villegas and Zaphiropoulos, 2015; Zhang et al., 2017a). Antisense lncRNA GLS-AS is dysregulated under nutrient stress which leads to MYC elevation and stabilization (Deng et al., 2019). The present results showed that Gm15441 negatively regulates Txnip expression during metabolic stress. LncRNA Gm15441 is antisense to Txnip, whose mRNA is decreased in liver coincident with the robust increase in Gm15441 
expression beginning 3 hours after activation of PPARA by WY-14643. The underlying PPARA regulatory interactions controlling activation of the $G m 15441$ promoter region were investigated, and four of seven PPARA binding sites identified within $10 \mathrm{~kb}$ of the Gm15441 TSS were confirmed by ChIP assays and shown to be functional in reporter gene assays. Thus, the TXNIP anti-sense lncRNA Gm15441 is a direct target of PPARA in WY-14643-stimulated mouse liver.

Pharmacological activation of PPARA deregulates NRLP3 inflammasome activity in a mouse model of diabetes (Deng et al., 2017) and prolonged hepatic NLRP3 inflammasome activation leads to hepatocyte death, inflammation, and fibrosis. Thus, the TXNIP-mediated NLRP3 inflammasome pathway was examined. Txnip mRNA was significantly decreased by PPARA activation in livers of wild-type, $G m 15441^{+/+}$mice, while TXNIP protein was elevated in livers of $G m 15441^{\mathrm{LSL}}$ mice. Furthermore, TXNIP-mediated NLRP3 inflammasome activation resulted in cleavage of CASP1 to its mature, proteolytically active form. Mature CASP1 subsequently cleaves target proteins including the proinflammatory cytokine IL1B to its corresponding mature, active form. Mature, cleaved CASP1 and IL1B protein were elevated in Gm $15441^{\mathrm{LSL}}$ mice, indicating an increase in inflammasome activation. These findings indicate that lncRNA Gm15441 suppresses the TXNIP-mediated NLRP3 inflammasome pathway in response to metabolic stress.

PPARA plays an important role in the fasted mouse liver model (Brocker et al., 2018). Hepatic expression of PPARA target genes Acadm and Cyp4a14 was significantly increased by fasting, as was the expression of Txnip mRNA, as was seen in livers of Gm $15441^{\mathrm{LSL}}$ mice as well as $G m 15441^{+/+}$mice compared with fed mice. TXNIP protein was more highly increased in fasting Gm $15441^{\mathrm{LSL}}$ mice compared to fasted $G m 15441^{+/+}$mice. Fasting Txnip mRNA levels are closely associated with lipid and glucose regulation (Szpigel et al., 2018; Waldhart et al., 2017). In addition, TXNIP-mediated NLRP3 inflammasome pathway target protein expression was significantly elevated in livers of fasting $G m 15441^{\mathrm{LSL}}$ mice compared to $\mathrm{Gm} 15441^{+/+}$mice. These results support the proposal that pharmacological and physiological upregulation of lncRNA Gm15441 prevents metabolic stress by suppressing the TXNIP-mediated NLRP3 inflammasome pathway.

PPARA, a key regulator of global lipid homeostasis, modulates fasting-induced lipid accumulation and hepatosteatosis in mice (Brocker et al., 2018). Lipid droplets appeared in livers of fasted $\mathrm{Gm} 15441^{+/+}$mice but were more widely distributed in livers of fasting $\mathrm{Gm} 15441^{\mathrm{LSL}}$ mice compared to fasting $G m 15441^{+/+}$mice. Histological lipid staining revealed considerably larger amounts of lipid accumulation in livers of fasted $\mathrm{Gm} 15441^{\mathrm{LSL}}$ mice than in fasted $\mathrm{Gm} 15441^{+/+}$ 
livers. Serum and liver TG levels and liver CHOL levels were significantly elevated by fasting in Gm $15441^{\text {LSL }}$ mouse livers. RNA-sequencing analysis revealed that liver damage, inflammation, and fatty accumulation biomarkers such as Apoa4, Bhmt, Lcn2, Orm2, Saa1, and Saa2 mRNAs showed significantly higher expression under fasting-induced metabolic stress in $G m 15441^{\mathrm{LSL}}$ compared to $\mathrm{Gm}_{15441^{+/+}}$mouse liver. LCN2 is marker for liver damage and inflammation (Asimakopoulou et al., 2016; Moschen et al., 2017). Hepatic Lcn2 and Orm2 mRNAs are both upregulated in the liver in response to IL1B (Sai et al., 2014; Zhang et al., 2014). Saal and Saa2 mRNAs are acute phase response proteins and biomarkers of inflammation and their protein products become major components high density lipoprotein (HDL) regulated by IL1B (Leclerc et al., 2019; Lindhorst et al., 1997; Olteanu et al., 2014). Moreover, SAA1 and SAA2 potentiate NLRP3 inflammasome activation, which would act as a feed forward much further increasing IL1B cleavage/maturation/inflammation (Chiba et al., 2009; Zhou et al., 2016). APOA4 plays a role in hepatic TG secretion and is upregulated in response to lipid accumulation (Qin et al., 2016; Zhang et al., 2017b). Notably, APOA4 is a regulator of fasting lipid that is associated with TG secretion and HDL cholesterol in type 2 diabetes (Delgado-Lista et al., 2010; Qi et al., 2007). APOA4 was stimulated by inflammation through activation of TNFR2 and NF-kB signaling in kidney tubular cells (Lee et al., 2017). BHMT remethylates homocysteine to methionine using betaine and contributes to methionine, homocysteine, S-adenosylmethionine, and glutathione homeostasis (Perez-Miguelsanz et al., 2017). In hepatocytes, BHMT upregulation prevent ER stress response, lipid accumulation, and cell death (Ji et al., 2007; Ji et al., 2008). Additionally, BHMT was identified as blood marker for acute liver injury and tumoral liver (Ma et al., 2014). The increase in mature/cleaved IL1B in $G m 15441^{\mathrm{LSL}}$ mice may at least partially explain the increased expression of Lcn2, Orm2, Saa1 and Saa2 mRNAs by fasting in Gm15441 ${ }^{\mathrm{LSL}}$ mouse liver. Taken together, these genes are all associated with inflammation/oxidative stress, consistent with the PPARA-dependent attenuation by Gm15441 of TXNIP-associated hepatic inflammation in response to metabolic stress.

An internal ribosome entry site (IRES) is an RNA element that allows for translation initiation (Lampe et al., 2018). Deregulation of IRES-mediated p53 translation promoted the defective p53 response against DNA damage in human cancer cells (Halaby et al., 2015). ER stress, serum deprivation, and hypoxia-induced human acetyl-CoA carboxylase 1 (hACC1) are controlled by IRES sequences (Damiano et al., 2018). Beclin-1 independent autophagy is promoted through IRES-dependent translation of hypoxia-inducible factor $1 \alpha$ (HIF $1 \alpha)$ (Wu et al., 2014). Polypyrimidine tract binding proteins (PTBs) were shown to regulate TXNIP expression 
during nutrition starvation by binding to IRES sequences within the Txnip 5'UTR (Lampe et al., 2018). By binding to these IRES sequences, PTB prevents translation initiation and suppresses TXNIP protein expression. As lncRNA Gm15441 is antisense to TXNIP and its exon 3 almost entirely overlaps the Txnip 5'UTR, Gm15441 may block IRES sequences needed for translation of TXNIP. The ability of Gm15441 to modulate TXNIP protein levels via Txnip 5'UTR IRES sequences was demonstrated in vitro by co-transfection of a GFP reporter with a Gm15441 expression vector. A decrease in GFP expression was dependent on both Gm15441 mRNA and the Txnip 5'UTR sequence, indicating that Gm15441 in part regulates TXNIP by binding to its 5'UTR, presumably by masking functional IRES sites.

A strand-specific Gm15441 knockout mouse was developed to assess lncRNA function without impacting regulation of its antisense protein-coding partner Txnip. In contrast, previous knockout mouse models developed to evaluate TXNIP function disrupt both Txnip and Gm15441 and therefore abolish the unique regulatory loop that exists between these two genes. Txnip-null mice show a higher incidence of hepatocellular carcinoma, with approximately $40 \%$ of male mice developing hepatic tumors (Kwon et al., 2010; Sheth et al., 2006). However, the Txnip-null mice lack exons 1-4, and thus Gm15441 is also ablated (Oka et al., 2006). Conditional Txnip-floxed mice were developed that target exon 1, which results in deletion of Gm15441 exon 3 (Oka et al., 2009). However, in another study, the ubiquitously-expressed protamine-Cre mouse was employed to generate a Txnip conditional-null mouse line (Yoshioka et al., 2007). Hepatocytespecific Txnip-null mice also used an exon 1 floxed allele which results in the removal of Gm15441 exon 2 (Chutkow et al., 2008). TXNIP expression in vitro inhibited hepatocellular carcinoma cell proliferation and induced apoptosis (Liu et al., 2017). Thus, prolonged Gm15441-mediated suppression of TXNIP may contribute to PPARA agonist induced hepatocellular carcinoma (Cho et al., 2019; Gunes et al., 2018).

In summary, the present study focused on the role of lncRNA as a potential metabolic regulator. Pharmacological and physiological activation of PPARA promoted induction of large numbers of hepatic lncRNAs, a subset of which are also targeted by the nuclear receptors CAR and PXR. Gm15441 was identified as a liver-specific, PPARA-dependent lncRNA positioned antisense to the pro-inflammatory gene Txnip. Gm15441 expression was shown to suppress TXNIP protein by a mechanism involving the blocking of IRES sites within its 5'UTR. By suppressing TXNIP translation, Gm15441 inhibits TXNIP-mediated activation of the NLRP3 inflammasome as well as subsequent CASP1 cleavage and IL1B maturation. Taken together, these 
results indicate that lncRNA Gm15441 directly prevents metabolic stress-induced inflammation and represents a novel therapeutic target for the treatment of inflammatory disorders.

\section{Author contributions}

C.N.B. and D.K. conducted the experiments and performed data analysis. C.N.B., D.J.W., and F.J.G. designed the experiments and wrote the paper. C.N.B. and D.K. performed all the mouse experiments. T.M., K.K. and D.J.W. analyzed NGS data and identified WY-14643-responsive liver lncRNAs, and D.J.W. revised and edited the manuscript. T.J.V., S.T. and J.A.B. contributed to the analysis of mouse experimental data. C.N.B. and F.J.G. supervised and coordinated the overall study.

\section{Acknowledgements}

We thank Linda G. Byrd and John R. Buckley for technical assistance with mouse studies. This work was funded by the intramural research program at the National Cancer Institute, National Institutes of Health and by NIH grant R01-ES024421 to D.J.W. C.N.B. was supported in part by the Postdoctoral Research Associate Training (PRAT) program through the National Institute of General Medical Sciences, National Institutes of Health. D.K. was supported in part by a grant from the Korea Health Technology R\&D Project through the Korea Health Industry Development Institute, funded by the Ministry of Health \& Welfare, Republic of Korea (HI17C2082). The content of this paper is solely the responsibility of the authors and does not necessarily represent the official views of the National Institutes of Health or Boston University. The Alb-Cre mice were provided by Derek LeRoith (Mount Sinai School of Medicine).

\section{Declaration of Interests}

The authors declare no conflicts of interest.

\section{CONTACT FOR REAGENT AND RESOURCE SHARING}

Further information and requests for resources and reagents should be directed to and will be fulfilled by the lead contact, Frank J. Gonzalez (gonzalef@mail.nih.gov).

\section{EXPERIMENTAL MODEL AND SUBJECT DETAILS}




\section{Mouse models}

Male 6- to 12-week-old mice were used for all studies and all mouse strains were on the C57BL/6J background and maintained on a grain-based control diet (NIH-31). Mice were housed in light and temperature-controlled rooms and were provided with water and pelleted chow ad libitum. For pharmacological studies, the mice were provided a grain-based control diet or matched diet containing $0.1 \%$ Wy-14643 for 24 or 48 hours. For monitoring the time dependence of gene responses, Wy-14643 was dissolved in 1\% carboxymethyl cellulose (CMC) solution and orally administered $(50 \mathrm{mg} / \mathrm{kg}$ in $200 \mu \mathrm{l})$ for indicated time points. At the end of the treatment period, the mice were killed by $\mathrm{CO}_{2}$ asphyxiation and tissues harvested. For physiological studies, food was removed for $24 \mathrm{~h}$ starting shortly after the onset of the light cycle and endpoints collected at the same time the following day. Animals were then killed, and tissue samples harvested for further analysis. Blood was collected by venipuncture of the caudal vena cava. All animal experiments were performed in accordance with the Association for Assessment and Accreditation of Laboratory Animal Care international guidelines and approved by the National Cancer Institute Animal Care and Use Committee.

\section{METHOD DETAILS}

\section{Generation of Gm15441-null mice}

SAGE Laboratories (Cambridge, UK) provided design and construction services for the CRISPR/Cas gene targeting technologies used to create a Gm15441-null mouse line. The targeting strategy results in the insertion of a floxed cassette containing a transcriptional stop repeat within the first intron of Gm15441 (NR_040409.1) (Table S3). Presence of the cassette prevents Gm15441 expression. Crossing with a Cre mouse line removes the stop cassette and allows Gm15441 expression to proceed. Microinjection-ready sgRNA, Cas 9 mRNA, and a plasmid donor with a floxed stop cassette were purchased from SAGE Laboratories. The sgRNA, Cas9 mRNA, and plasmid donor were then injected into C57BL/6J mouse embryos by the Transgenic Mouse Model Laboratory at the National Cancer Institute (Fredrick, MD) using the manufacturer's recommended protocol. Founder animals were genotyped using primer sets in Table S4, and all modifications confirmed by targeted sequencing. Homozygous mice were then backcrossed ten times into the C57BL6 background bred out any off-target effects.

\section{Mouse genotyping}


Genomic DNA was extracted from tail using extraction E buffer, TPS and N buffer. $25 \mu 1$ of E buffer and $7 \mathrm{ul}$ of TPS buffer were added and stored room temperature at $10 \mathrm{~min}$. Then tails were heated at $95^{\circ} \mathrm{C}$ in $5 \mathrm{~min}$ and $25 \mu \mathrm{l}$ of $\mathrm{N}$ buffer was added. Polymerase chain reaction was used for examined of genotype using the following primers: forward: 5'TGCGAGGCACGATATGGCGA-3', reverse: 5'-AGCGCACCTGTCACTTTCCTGC-3'. The amplicon with 1200 and 1700 bp were detected in wild-type and Gm15441-null mice, respectively.

\section{RNA isolation, cDNA library construction, and sequencing}

RNeasy Plus Mini Kit (Qiagen, Valencia, CA, USA) was used to extract total RNA from livers from four different treatment and control groups: wild-type mice and Ppara $^{-/-}$mice fed either control diet or fed a diet containing 0.1\% WY-14643 for 48 hours and euthanized between 1 and 3 PM. RNAs were extracted from $\mathrm{n}=9$ to 15 independent livers per group, and after quality assessment of RNA by TapeStation 4200 (Agilent, Santa Clara, CA, USA), high quality RNA samples (RIN>9.0) were pooled, as follows, then used to construct stranded RNA-seq libraries from polyA-selected total liver RNA using an Illumina stranded TruSeq mRNA Prep Kit (Illumina, San Diego, CA, USA). Three independent RNA pools were prepared for each of the 4 treatment groups, with each pool comprised of $\mathrm{n}=3,4$ or 5 individual liver RNA samples. The libraries were subjected to 126 cycle paired-end sequencing using an Illumina HiSeq 2500 instrument (Illumina) at the NCI-CCR sequencing facility (Frederic, USA) at a depth of 30-42 million read pairs for each of the 12 RNA-seq libraries. For RNA-seq analysis of $G m 15441^{\mathrm{LSL}}$ mice, total liver RNA was isolated from wild-type mice and $G m 15441^{\mathrm{LSL}}$ mice fed either control diet or a diet containing 0.1\% WY-14643 for 24 hours and killed between 1 and 3 PM. RNAs were extracted from n =912 independent livers per experimental group, analyzed and used to prepare sequencing libraries as described above. Three independent RNA pools were prepared for each of the 4 treatment groups, with each pool comprised of $n=2-3$ individual liver RNA samples. Libraries were subjected to 150 cycle paired-end Illumina sequencing at a depth of 13-21 million read pairs for each of the 12 RNA-seq libraries.

\section{Analysis of sequencing results}

Data were analyzed using a custom RNA-seq analysis pipeline (Connerney et al., 2017) as described elsewhere (Lodato et al., 2017). Briefly, sequence reads were mapped to the mouse genome (release mm9) using TopHat2 (v2.1.1) (Kim et al., 2013). Genomic regions that contain exonic sequence in at least one isoform of a gene (exon collapsed regions; (Connerney et al., 2017)) 
were defined for each RefSeq gene and for each lncRNA gene. HTSeq (0.6.1p1) was then used to obtain read counts for exon collapsed regions of RefSeq genes, and featureCounts (1.4.6-p5) was used to obtain read counts for exon collapsed regions of lncRNA genes. A set of 24,197 annotated mouse RefSeq genes (which includes some RefSeq lncRNAs) and a set of 15,558 liver-expressed lncRNA genes (Lodato et al., 2017; Melia and Waxman, 2019) was considered for differential expression analysis. These lncRNAs include intergenic lncRNAs, as well as lncRNAs that are antisense or intragenic with respect to RefSeq genes, and were discovered using a computational pipeline for lncRNA discovery described elsewhere (Melia et al., 2016) based on 186 mouse liver RNA-seq datasets representing 30 different biological conditions. RefSeq and lncRNA genes that showed significant differential expression following exposure to WY-14643 were identified by EdgeR as outlined elsewhere (Melia et al., 2016). Genes dysregulated with an expression foldchange (i.e., either up regulation or down regulation) $>2$ at a false discovery rate (FDR), i.e., an adjusted P-value $<0.05$ were considered significant and are shown in Table S1 and Table S2. Raw and processed RNA-seq data are available at GEO (https:/www.ncbi.nlm.nih.gov/gds) accession numbers GSE132385 and GSE132386.

\section{Cell culture}

Primary hepatocytes were isolated from C57BL6N mice as previously reported (Seglen, 1976) and seeded on collagen-coated 12-well plates (Becton Dickinson and Company, Franklin Lakes, NJ) at a density of $2 \times 10^{5}$ cells in Williams' Medium E (Thermo-Fisher Scientific, Waltham, MA) supplemented with 5\% FBS and penicillin/streptomycin/amphotericin B solution (Gemini Bioproducts, West Sacramento, CA). Hepa-1 mouse hepatoma cells and NIHT3T mouse embryonic fibroblast cells (ATCC, Manassas, VA) were maintained at $37^{\circ} \mathrm{C}$ in a humidified atmosphere of 5\% $\mathrm{CO}_{2}$ in Dulbecco's Modified Eagle Medium (DMEM) containing 10\% Fetal Bovine Serum (FBS) and 1\% of penicillin/streptomycin mixture (Invitrogen, Waltham, MA).

\section{Histological staining}

Fresh liver tissue was immediately fixed in 10\% phosphate-buffered formalin for $24 \mathrm{~h}$ and then processed in paraffin blocks. Four-micrometer sections were used for H\&E staining. Sections were processed by HistoServ, Inc. (Germantown, MD). Slide imaging was performed using a Keyence BZ-X700 series all-in-one microscope with both $20 \times$ and $40 \times$ objectives, $200 \times$ and $400 \times$ magnification, respectively. 


\section{Serum biochemistry}

Blood was collected from mice and transferred to BD Microtainer Serum Separator Tubes (Becton Dickinson, Franklin Lakes, NJ). Serum was flash frozen in liquid nitrogen and stored at -80C. Serum chemistry analysis for total cholesterol (CHOL), TG was performed using Wako Clinical Diagnostics kits (WakoUSA, Richmond, VA). Serum alanine aminotransferase (ALT) and aspartate aminotransferase (AST) levels were measured using Catachem VETSPEC Kits as recommended by the manufacturer (Catachem, Oxford, CT). Blood GLU levels were measured using a Contour blood GLU meter (Bayer, Mishawaka, IN).

\section{Western blot analysis}

Whole cell extracts were prepared from mouse liver tissue or mouse primary hepatocytes using RIPA buffer supplemented with Halt Protease and Phosphatase Inhibitor Cocktail (Thermo-Fisher Scientific) and $1 \mathrm{mM}$ PMSF. Protein concentrations were determined using the Pierce BCA Protein Assay Kit (Pierce, Rockford, IL). Forty $\mu \mathrm{g}$ of protein was loaded per lane on a 4-12\% Criterion TGX Precast Gel (Bio-Rad) then transferred to PVDF membranes using a Trans-Blot Turbo Transfer System (Bio-Rad). Membranes were blocked in 5\% nonfat milk followed by an overnight incubation with primary antibodies targeting TXNIP (Novus; NBP1-54578), CASP1 (Proteintech; 22915-1-AP), IL1B (Cell Signaling; \#12242), or TUBA1B (Epitomics; 1878-1) at $4^{\circ} \mathrm{C}$. Following primary antibody incubation, the blots were washed and incubated with HRPconjugated secondary antibodies for one hour (Cell Signaling; \#7074S, \#7076S). The blots were then stripped using Restore Western Blot Stripping Buffer (Thermo-Fisher Scientific) and reprobed with alternate antibodies. An antibody against ACTB (Cell Signaling; 8457L) was used as a loading control. Blot imaging was performed on a ChemiDoc MP System (Bio-Rad) after exposing the blot to Clarity Western ECL Blotting Substrate (Bio-Rad). Protein expression was quantitatively analyzed using band density and ImageJ software (NIH, Bethesda MD) (Schneider et al., 2012).

\section{Quantitative reverse transcription PCR assays}

Total RNA was isolated from fresh mouse liver, mouse primary hepatocytes, and Hepa-1 cells using TRIzol Reagent (Thermo-Fisher Scientific, Waltham, MA, USA) and quantified using a NanoDrop Spectrophotometer (NanoDrop Products, Wilmington, DE, USA). Total RNA (2 $\mu \mathrm{g})$ was reverse transcribed using All-in-One cDNA Synthesis SuperMix (BioTool, Houston, TX, 
USA). qRT-PCR analysis was performed using SYBR Green qPCR Master Mix (BioTool). Primers were designed for gene specificity and to cross exon-exon junctions using Primer-BLAST (www.ncbi.nlm.nih.gov/tools/primer-blast/) and purchased from IDT DNA Technologies (Coralville, IA, USA) (Table S4). qRT-PCR experiments were designed and performed according to Minimum Information for Publication of Quantitative Real-Time PCR Experiments (MIQE) guidelines (Plain et al., 2014). Results are normalized to actin expression. Values given are fold over control or relative expression value, where appropriate, calculated using the $2 \Delta \mathrm{Ct} \mathrm{QPCR}$ calculation method (Pfaffl, 2001).

\section{Luciferase reporter assays}

For luciferase assays, pSG5-PPARA (mouse) and pSG5-RXRA (mouse) were used for transcription factor expression (Shah et al., 2007). Custom GeneBlocks (IDT DNA) were synthesized containing the predicted PPRE sites for Gm15441. GeneBlocks were digested and purified using a Qiagen PCR Purification Kit (Qiagen, Valencia, CA), and cloned into the pGL4.11 for PPRE reporter constructs (Promega, Madison, WI) using a BioRad Quick Ligation Kit (BioRad, Hercules, CA, USA). Prior to performing assays, all constructs were confirmed by Sanger sequencing at the NCI Center for Cancer Research Genomics Core. The phRL-TK renilla luciferase construct was used as a control to normalize for transfection efficiency. Primary hepatocytes were seeded into 12 -well plates $\left(4 \times 10^{4}\right.$ cells/well). PPRE reporter constructs were co-transfected into hepatocytes with PPARA and RXR expression. vectors. PPRE-luc plasmid containing an Acox1 PPRE site repeat was used as a positive control (Kliewer et al., 1992). Empty pGL4.11 plasmid was used as negative controls. Plasmids were transfected using Lipofectamine 3000 Reagent (Thermo-Fisher Scientific). Luciferase activities were measured and plotted relative to lysate protein concentrations using the Promega Dual Luciferase Reporter (Promega) assays according to the manufacturer's protocol. Measurements were taken on a Veritas microplate luminometer (Turner Biosystems, Sunnyvale, CA, USA).

\section{Chromatin immunoprecipitation}

Chromatin was prepared from hepatocytes for ChIP assays as previously described (Kim et al., 2014). Cells were fixed with $4 \%$ paraformaldehyde for $15 \mathrm{~min}$, then glycine was added to a final concentration of $0.125 \mathrm{M}$ and incubated for $10 \mathrm{~min}$ before harvesting. Chromatin was sonicated using a Bioruptor Pico (Diagenode, Denville, NJ, USA). Chromatin preparations were subjected to ChIP using a ChIP-IT High Sensitivity Kit and Protein G Agarose Prepacked Columns (Active 
Motif, Carlsbad, CA, USA) using either PPARA (Abcam; Ab24509) antibody. Normal rabbit IgG (Cell Signaling Technologies; \#2729S) and Histone H3 (Cell Signaling Technologies; \#4620) antibody were used as negative and positive controls, respectively. DNA was purified and concentrated using MinElute Reaction Cleanup columns (Qiagen). qRT-PCR and conventional PCR were performed using $2 \mu \mathrm{l}$ of ChIP DNA samples from the $50 \mu$ of purified samples using gene-specific primers (Table S4). Cycle threshold $(\mathrm{Ct})$ values of ChIP and input samples were calculated and presented as fold change.

\section{RNA fluorescent in situ (FISH) hybridization}

For fluorescence in situ hybridization (FISH) staining of $\mathrm{Gm} 15441, \mathrm{Gm}_{15441^{+/+}}$and $\mathrm{Gm} 15441^{\mathrm{LSL}}$ mice were fed either a control diet or diet containing WY-14643 for 36 hours. Livers were harvested, fixed in $4 \%$ of paraformaldehyde overnight then sent to the Molecular Pathology Laboratory (PHL) at the National Cancer Institute for processing. RNA FISH experiments were performed by the using custom RNAscope probes and reagents developed by Advanced Cell Diagnostics (Newark, CA). Proprietary FISH probes targeted a region of Gm15441 that does not overlap with Txnip to prevent signal interference (Table S5). Slide imaging was performed using Aperio ImageScope software (Leica Biosystems, Buffalo Grove, IL, USA).

\section{QUANTIFICATION AND STATISTICAL ANALYSIS}

PPARA ChIP-seq data was downloaded from NCBI Gene Expression Omnibus (GSE61817). CHIP-seq data was uploaded to the Galaxy public server at usegalaxy.org to analyze the data (Afgan et al., 2018). More specifically, data were converted to bigwig file format using Galaxy tools. Bigwig files were then visualized using Integrated Genome Browser (version 9.0.0) (Freese et al., 2016). All results are expressed as means \pm SD. Significance was determined by t-test or one-way ANOVA with Bonferroni correction using Prism 7.0 software (GraphPad Software, La Jolla, CA, USA). A P value less than 0.05 was considered significant and statistical significance is indicated in the figure legends. 


\section{References}

Abcouwer, S.F. (2013). Direct effects of PPAR $\alpha$ agonists on retinal inflammation and angiogenesis may explain how fenofibrate lowers risk of severe proliferative diabetic retinopathy. Diabetes 62, 36-38.

Afgan, E., Baker, D., Batut, B., van den Beek, M., Bouvier, D., Cech, M., Chilton, J., Clements, D., Coraor, N., Gruning, B.A., et al. (2018). The Galaxy platform for accessible, reproducible and collaborative biomedical analyses: 2018 update. Nucleic Acids Res 46, W537-W544.

Andrejeva, G., and Rathmell, J.C. (2017). Similarities and distinctions of cancer and immune metabolism in inflammation and tumors. Cell Metab 26, 49-70.

Anthony, T.G., and Wek, R.C. (2012). TXNIP switches tracks toward a terminal UPR. Cell Metab 16, 135-137.

Asimakopoulou, A., Weiskirchen, S., and Weiskirchen, R. (2016). Lipocalin 2 (LCN2) expression in hepatic malfunction and therapy. Front Physiol 7, 430.

Bai, R.X., Xu, Y.Y., Qin, G., Chen, Y.M., Wang, H.F., Wang, M., and Du, S.Y. (2019). Repression of TXNIP-NLRP3 axis restores intestinal barrier function via inhibition of myeloperoxidase activity and oxidative stress in nonalcoholic steatohepatitis. J Cell Physiol 234, 7524-7538.

Bettencourt, I.A., and Powell, J.D. (2017). Targeting metabolism as a novel therapeutic approach to autoimmunity, inflammation, and transplantation. J Immunol 198, 999-1005.

Brocker, C.N., Patel, D.P., Velenosi, T.J., Kim, D., Yan, T., Yue, J., Li, G., Krausz, K.W., and Gonzalez, F.J. (2018). Extrahepatic PPAR $\alpha$ modulates fatty acid oxidation and attenuates fasting-induced hepatosteatosis in mice. J Lipid Res 59, 2140-2152.

Chiba, T., Han, C.Y., Vaisar, T., Shimokado, K., Kargi, A., Chen, M.H., Wang, S., McDonald, T.O., O'Brien, K.D., Heinecke, J.W., et al. (2009). Serum amyloid A3 does not contribute to circulating SAA levels. J Lipid Res 50, 1353-1362.

Cho, S.Y., Kim, S., Son, M.J., Rou, W.S., Kim, S.H., Eun, H.S., and Lee, B.S. (2019). Clinical significance of the thioredoxin system and thioredoxin-domain-containing protein family in hepatocellular carcinoma. Dig Dis Sci 64, 123-136.

Chutkow, W.A., Patwari, P., Yoshioka, J., and Lee, R.T. (2008). Thioredoxin-interacting protein (Txnip) is a critical regulator of hepatic glucose production. J Biol Chem 283, 2397-2406.

Connerney, J., Lau-Corona, D., Rampersaud, A., and Waxman, D.J. (2017). Activation of male liver chromatin accessibility and STAT5-dependent gene transcription by plasma growth Hormone Pulses. Endocrinology 158, 1386-1405.

Corrales, P., Vivas, Y., Izquierdo-Lahuerta, A., Horrillo, D., Seoane-Collazo, P., Velasco, I., Torres, L., Lopez, Y., Martinez, C., Lopez, M., et al. (2019). Long-term caloric restriction 
ameliorates deleterious effects of aging on white and brown adipose tissue plasticity. Aging Cell, e12948.

Cui, J.Y., and Klaassen, C.D. (2016). RNA-Seq reveals common and unique PXR- and CARtarget gene signatures in the mouse liver transcriptome. Biochim Biophys Acta 1859, 1198-1217.

Dallner, O.S., Marinis, J.M., Lu, Y.H., Birsoy, K., Werner, E., Fayzikhodjaeva, G., Dill, B.D., Molina, H., Moscati, A., Kutalik, Z., et al. (2019). Dysregulation of a long noncoding RNA reduces leptin leading to a leptin-responsive form of obesity. Nat Med 25, 507-516.

Damiano, F., Testini, M., Tocci, R., Gnoni, G.V., and Siculella, L. (2018). Translational control of human acetyl-CoA carboxylase $1 \mathrm{mRNA}$ is mediated by an internal ribosome entry site in response to ER stress, serum deprivation or hypoxia mimetic $\mathrm{CoCl}_{2}$. Biochim Biophys Acta $\mathrm{Mol}$ Cell Biol Lipids 1863, 388-398.

Delgado-Lista, J., Perez-Jimenez, F., Ruano, J., Perez-Martinez, P., Fuentes, F., Criado-Garcia, J., Parnell, L.D., Garcia-Rios, A., Ordovas, J.M., and Lopez-Miranda, J. (2010). Effects of variations in the APOA1/C3/A4/A5 gene cluster on different parameters of postprandial lipid metabolism in healthy young men. J Lipid Res 51, 63-73.

Deng, S.J., Chen, H.Y., Zeng, Z., Deng, S., Zhu, S., Ye, Z., He, C., Liu, M.L., Huang, K., Zhong, J.X., et al. (2019). Nutrient stress-dysregulated antisense lncRNA GLS-AS impairs GLSmediated metabolism and represses pancreatic cancer progression. Cancer Res 79, 1398-1412.

Deng, Y., Han, X., Yao, Z., Sun, Y., Yu, J., Cai, J., Ren, G., Jiang, G., and Han, F. (2017). PPAR $\alpha$ agonist stimulated angiogenesis by improving endothelial precursor cell function via a NLRP3 inflammasome pathway. Cell Physiol Biochem 42, 2255-2266.

Forman, B.M., Chen, J., and Evans, R.M. (1997). Hypolipidemic drugs, polyunsaturated fatty acids, and eicosanoids are ligands for peroxisome proliferator-activated receptors $\alpha$ and $\delta$. Proc Natl Acad Sci U S A 94, 4312-4317.

Freese, N.H., Norris, D.C., and Loraine, A.E. (2016). Integrated genome browser: visual analytics platform for genomics. Bioinformatics 32, 2089-2095.

Gonzalez, F.J., Fang, Z.Z., and Ma, X. (2015). Transgenic mice and metabolomics for study of hepatic xenobiotic metabolism and toxicity. Expert Opin Drug Metab Toxicol 11, 869-881.

Goodpaster, B.H., and Sparks, L.M. (2017). Metabolic flexibility in health and disease. Cell Metab 25, 1027-1036.

Guil, S., and Esteller, M. (2012). Cis-acting noncoding RNAs: friends and foes. Nat Struct Mol Biol 19, 1068-1075.

Gunes, A., Bagirsakci, E., Iscan, E., Cakan-Akdogan, G., Aykutlu, U., Senturk, S., Ozhan, G., Erdal, E., Nart, D., Barbet, F.Y., et al. (2018). Thioredoxin interacting protein promotes invasion in hepatocellular carcinoma. Oncotarget 9, 36849-36866. 
Halaby, M.J., Harris, B.R., Miskimins, W.K., Cleary, M.P., and Yang, D.Q. (2015). Deregulation of internal ribosome entry site-mediated p53 translation in cancer cells with defective p53 response to DNA damage. Mol Cell Biol 35, 4006-4017.

Hong, K., Xu, G., Grayson, T.B., and Shalev, A. (2016). Cytokines regulate $\beta$-cell thioredoxininteracting protein (TXNIP) via distinct mechanisms and pathways. J Biol Chem 291, 84288439.

Iwakiri, J., Terai, G., and Hamada, M. (2017). Computational prediction of lncRNA-mRNA interactionsby integrating tissue specificity in human transcriptome. Biol Direct 12, 15.

Jandl, K., Thekkekara Puthenparampil, H., Marsh, L.M., Hoffmann, J., Wilhelm, J., Veith, C., Sinn, K., Klepetko, W., Olschewski, H., Olschewski, A., et al. (2019). Long non-coding RNAs influence the transcriptome in pulmonary arterial hypertension: the role of PAXIP1-AS1. J Pathol 247, 357-370.

Ji, C., Shinohara, M., Kuhlenkamp, J., Chan, C., and Kaplowitz, N. (2007). Mechanisms of protection by the betaine-homocysteine methyltransferase/betaine system in HepG2 cells and primary mouse hepatocytes. Hepatology 46, 1586-1596.

Ji, C., Shinohara, M., Vance, D., Than, T.A., Ookhtens, M., Chan, C., and Kaplowitz, N. (2008). Effect of transgenic extrahepatic expression of betaine-homocysteine methyltransferase on alcohol or homocysteine-induced fatty liver. Alcohol Clin Exp Res 32, 1049-1058.

Kim, D., Pertea, G., Trapnell, C., Pimentel, H., Kelley, R., and Salzberg, S.L. (2013). TopHat2: accurate alignment of transcriptomes in the presence of insertions, deletions and gene fusions. Genome Biol 14, R36.

Kim, J.H., Qu, A., Reddy, J.K., Gao, B., and Gonzalez, F.J. (2014). Hepatic oxidative stress activates the Gadd 45 bene by way of degradation of the transcriptional repressor STAT3. Hepatology 59, 695-704.

Kim, S.K., Choe, J.Y., and Park, K.Y. (2019). TXNIP-mediated nuclear factor-kappaB signaling pathway and intracellular shifting of TXNIP in uric acid-induced NLRP3 inflammasome. Biochem Biophys Res Commun 511, 725-731.

Kliewer, S.A., Sundseth, S.S., Jones, S.A., Brown, P.J., Wisely, G.B., Koble, C.S., Devchand, P., Wahli, W., Willson, T.M., Lenhard, J.M., et al. (1997). Fatty acids and eicosanoids regulate gene expression through direct interactions with peroxisome proliferator-activated receptors $\alpha$ and $\gamma$. Proc Natl Acad Sci U S A 94, 4318-4323.

Kliewer, S.A., Umesono, K., Noonan, D.J., Heyman, R.A., and Evans, R.M. (1992). Convergence of 9-cis retinoic acid and peroxisome proliferator signalling pathways through heterodimer formation of their receptors. Nature 358, 771-774.

Kono, K., Kamijo, Y., Hora, K., Takahashi, K., Higuchi, M., Kiyosawa, K., Shigematsu, H., Gonzalez, F.J., and Aoyama, T. (2009). PPAR $\alpha$ attenuates the proinflammatory response in activated mesangial cells. Am J Physiol Renal Physiol 296, F328-336. 
Krysiak, R., Gdula-Dymek, A., and Okopien, B. (2011). Effect of simvastatin and fenofibrate on cytokine release and systemic inflammation in type 2 diabetes mellitus with mixed dyslipidemia. Am J Cardiol 107, 1010-1018 e1011.

Kwon, H.J., Won, Y.S., Suh, H.W., Jeon, J.H., Shao, Y., Yoon, S.R., Chung, J.W., Kim, T.D., Kim, H.M., Nam, K.H., et al. (2010). Vitamin D3 upregulated protein 1 suppresses TNF-alphainduced NF-kappaB activation in hepatocarcinogenesis. J Immunol 185, 3980-3989.

Lampe, S., Kunze, M., Scholz, A., Brauss, T.F., Winslow, S., Simm, S., Keller, M., Heidler, J., Wittig, I., Brune, B., et al. (2018). Identification of the TXNIP IRES and characterization of the impact of regulatory IRES trans-acting factors. Biochim Biophys Acta Gene Regul Mech 1861, 147-157.

Leclerc, D., Christensen, K.E., Cauvi, O., Yang, E., Fournelle, F., Bahous, R.H., Malysheva, O.V., Deng, L., Wu, Q., Zhou, Z., et al. (2019). Mild methylenetetrahydrofolate reductase deficiency alters inflammatory and lipid pathways in liver. Mol Nutr Food Res 63, e1801001.

Lee, H.H., Cho, Y.I., Kim, S.Y., Yoon, Y.E., Kim, K.S., Hong, S.J., and Han, W.K. (2017). TNF- $\alpha$-induced Inflammation stimulates apolipoprotein-A4 via activation of TNFR2 and NFkappaB signaling in kidney tubular cells. Sci Rep 7, 8856.

Lee, J.M., Wagner, M., Xiao, R., Kim, K.H., Feng, D., Lazar, M.A., and Moore, D.D. (2014a). Nutrient-sensing nuclear receptors coordinate autophagy. Nature 516, 112-115.

Lee, J.W., Bajwa, P.J., Carson, M.J., Jeske, D.R., Cong, Y., Elson, C.O., Lytle, C., and Straus, D.S. (2007). Fenofibrate represses interleukin-17 and interferon- $\alpha$ expression and improves colitis in interleukin-10-deficient mice. Gastroenterology 133, 108-123.

Lee, S., Min Kim, S., Dotimas, J., Li, L., Feener, E.P., Baldus, S., Myers, R.B., Chutkow, W.A., Patwari, P., Yoshioka, J., et al. (2014b). Thioredoxin-interacting protein regulates protein disulfide isomerases and endoplasmic reticulum stress. EMBO Mol Med 6, 732-743.

Lerner, A.G., Upton, J.P., Praveen, P.V., Ghosh, R., Nakagawa, Y., Igbaria, A., Shen, S., Nguyen, V., Backes, B.J., Heiman, M., et al. (2012). IRE1 $\alpha$ induces thioredoxin-interacting protein to activate the NLRP3 inflammasome and promote programmed cell death under irremediable ER stress. Cell Metab 16, 250-264.

Li, J., Yue, Z., Xiong, W., Sun, P., You, K., and Wang, J. (2017a). TXNIP overexpression suppresses proliferation and induces apoptosis in SMMC7221 cells through ROS generation and MAPK pathway activation. Oncol Rep 37, 3369-3376.

Li, L., Ismael, S., Nasoohi, S., Sakata, K., Liao, F.F., McDonald, M.P., and Ishrat, T. (2019). Thioredoxin-interacting protein (TXNIP) associated NLRP3 inflammasome activation in human alzheimer's disease brain. J Alzheimers Dis 68, 255-265.

Li, Y., Li, S., Luo, Y., Liu, Y., and Yu, N. (2017b). LncRNA PVT1 regulates chondrocyte apoptosis in osteoarthritis by acting as a sponge for miR-488-3p. DNA Cell Biol 36, 571-580. 
Lindhorst, E., Young, D., Bagshaw, W., Hyland, M., and Kisilevsky, R. (1997). Acute inflammation, acute phase serum amyloid A and cholesterol metabolism in the mouse. Biochim Biophys Acta 1339, 143-154.

Liu, Y., Lou, G., Norton, J.T., Wang, C., Kandela, I., Tang, S., Shank, N.I., Gupta, P., Huang, M., Avram, M.J., et al. (2017). 6-Methoxyethylamino-numonafide inhibits hepatocellular carcinoma xenograft growth as a single agent and in combination with sorafenib. FASEB J 31 , 5453-5465.

Lodato, N.J., Melia, T., Rampersaud, A., and Waxman, D.J. (2017). Sex-differential responses of tumor promotion-associated genes and dysregulation of novel long noncoding RNAs in constitutive androstane receptor-activated mouse liver. Toxicol Sci 159, 25-41.

Ma, H., Ning, J., Jin, X., Mao, C., Bu, X., Wang, M., Liu, H., Wang, K., Lausted, C., Hood, L., et al. (2014). Betaine homocysteine methyltransferase (BHMT) as a specific and sensitive blood marker for acute liver injury. Biomarkers 19, 578-584.

Mandala, A., Das, N., Bhattacharjee, S., Mukherjee, B., Mukhopadhyay, S., and Roy, S.S. (2016). Thioredoxin interacting protein mediates lipid-induced impairment of glucose uptake in skeletal muscle. Biochem Biophys Res Commun 479, 933-939.

Marin-Bejar, O., Mas, A.M., Gonzalez, J., Martinez, D., Athie, A., Morales, X., Galduroz, M., Raimondi, I., Grossi, E., Guo, S., et al. (2017). The human lncRNA LINC-PINT inhibits tumor cell invasion through a highly conserved sequence element. Genome Biol 18, 202.

Melia, T., Hao, P., Yilmaz, F., and Waxman, D.J. (2016). Hepatic long intergenic noncoding RNAs: high promoter conservation and dynamic, sex-dependent transcriptional regulation by Growth Hormone. Mol Cell Biol 36, 50-69.

Melia, T., and Waxman, D.J. (2019). Sex-biased lncRNAs inversely correlate with sex-opposite gene coexpression networks in diversity outbred mouse liver. Endocrinology 160, 989-1007.

Militello, G., Hosen, M.R., Ponomareva, Y., Gellert, P., Weirick, T., John, D., Hindi, S.M., Mamchaoui, K., Mouly, V., Doring, C., et al. (2018). A novel long non-coding RNA Myolinc regulates myogenesis through TDP-43 and Filip1. J Mol Cell Biol 10, 102-117.

Mochizuki, K., Suruga, K., Fukami, H., Kiso, Y., Takase, S., and Goda, T. (2006). Selectivity of fatty acid ligands for PPAR $\alpha$ which correlates both with binding to cis-element and DNA binding-independent transactivity in Caco-2 cells. Life Sci 80, 140-145.

Montagner, A., Polizzi, A., Fouche, E., Ducheix, S., Lippi, Y., Lasserre, F., Barquissau, V., Regnier, M., Lukowicz, C., Benhamed, F., et al. (2016). Liver PPARalpha is crucial for wholebody fatty acid homeostasis and is protective against NAFLD. Gut 65, 1202-1214.

Moschen, A.R., Adolph, T.E., Gerner, R.R., Wieser, V., and Tilg, H. (2017). Lipocalin-2: A master mediator of intestinal and metabolic inflammation. Trends Endocrinol Metab 28, 388397.

Muoio, D.M. (2007). TXNIP links redox circuitry to glucose control. Cell Metab 5, 412-414. 
Oka, S., Liu, W., Masutani, H., Hirata, H., Shinkai, Y., Yamada, S., Yoshida, T., Nakamura, H., and Yodoi, J. (2006). Impaired fatty acid utilization in thioredoxin binding protein-2 (TBP-2)deficient mice: a unique animal model of Reye syndrome. FASEB J 20, 121-123.

Oka, S., Yoshihara, E., Bizen-Abe, A., Liu, W., Watanabe, M., Yodoi, J., and Masutani, H. (2009). Thioredoxin binding protein-2/thioredoxin-interacting protein is a critical regulator of insulin secretion and peroxisome proliferator-activated receptor function. Endocrinology 150, $1225-1234$.

Olteanu, S., Kandel-Kfir, M., Shaish, A., Almog, T., Shemesh, S., Barshack, I., Apte, R.N., Harats, D., and Kamari, Y. (2014). Lack of interleukin-1 $\alpha$ in kupffer cells attenuates liver inflammation and expression of inflammatory cytokines in hypercholesterolaemic mice. Dig Liver Dis 46, 433-439.

Oslowski, C.M., Hara, T., O'Sullivan-Murphy, B., Kanekura, K., Lu, S., Hara, M., Ishigaki, S., Zhu, L.J., Hayashi, E., Hui, S.T., et al. (2012). Thioredoxin-interacting protein mediates ER stress-induced $\beta$ cell death through initiation of the inflammasome. Cell Metab 16, 265-273.

Perez-Miguelsanz, J., Vallecillo, N., Garrido, F., Reytor, E., Perez-Sala, D., and Pajares, M.A. (2017). Betaine homocysteine S-methyltransferase emerges as a new player of the nuclear methionine cycle. Biochim Biophys Acta Mol Cell Res 1864, 1165-1182.

Perron, U., Provero, P., and Molineris, I. (2017). In silico prediction of lncRNA function using tissue specific and evolutionary conserved expression. BMC Bioinformatics 18, 144.

Pfaffl, M.W. (2001). A new mathematical model for relative quantification in real-time RT-PCR. Nucleic Acids Res 29, e45.

Plain, K.M., Marsh, I.B., Waldron, A.M., Galea, F., Whittington, A.M., Saunders, V.F., Begg, D.J., de Silva, K., Purdie, A.C., and Whittington, R.J. (2014). High-throughput direct fecal PCR assay for detection of mycobacterium avium subsp. paratuberculosis in sheep and cattle. J Clin Microbiol 52, 745-757.

Qi, L., Liu, S., Rifai, N., Hunter, D., and Hu, F.B. (2007). Associations of the apolipoprotein A1/C3/A4/A5 gene cluster with triglyceride and HDL cholesterol levels in women with type 2 diabetes. Atherosclerosis 192, 204-210.

Qin, W., Li, X., Xie, L., Li, S., Liu, J., Jia, L., Dong, X., Ren, X., Xiao, J., Yang, C., et al. (2016). A long non-coding RNA, APOA4-AS, regulates APOA4 expression depending on HuR in mice. Nucleic Acids Res 44, 6423-6433.

Qu, X., Alsager, S., Zhuo, Y., and Shan, B. (2019). HOX transcript antisense RNA (HOTAIR) in cancer. Cancer Lett 454, 90-97.

Ralston, J.C., Lyons, C.L., Kennedy, E.B., Kirwan, A.M., and Roche, H.M. (2017). Fatty acids and NLRP3 inflammasome-mediated inflammation in metabolic tissues. Annu Rev Nutr 37, $77-$ 102. 
Ringelhan, M., Pfister, D., O'Connor, T., Pikarsky, E., and Heikenwalder, M. (2018). The immunology of hepatocellular carcinoma. Nat Immunol 19, 222-232.

Sai, K., Kurose, K., Koizumi, T., Katori, N., Sawada, J., Matsumura, Y., Saijo, N., Yamamoto, N., Tamura, T., Okuda, H., et al. (2014). Distal promoter regions are responsible for differential regulation of human orosomucoid-1 and -2 gene expression and acute phase responses. Biol Pharm Bull 37, 164-168.

Schaefer, M.B., Pose, A., Ott, J., Hecker, M., Behnk, A., Schulz, R., Weissmann, N., Gunther, A., Seeger, W., and Mayer, K. (2008). Peroxisome proliferator-activated receptor- $\alpha$ reduces inflammation and vascular leakage in a murine model of acute lung injury. Eur Respir J 32, 1344-1353.

Schneider, C.A., Rasband, W.S., and Eliceiri, K.W. (2012). NIH Image to ImageJ: 25 years of image analysis. Nat Methods 9, 671-675.

Seglen, P.O. (1976). Preparation of isolated rat liver cells. Methods Cell Biol 13, 29-83.

Shah, Y.M., Morimura, K., Yang, Q., Tanabe, T., Takagi, M., and Gonzalez, F.J. (2007). Peroxisome proliferator-activated receptor $\alpha$ regulates a microRNA-mediated signaling cascade responsible for hepatocellular proliferation. Mol Cell Biol 27, 4238-4247.

Shen, L., Yang, Y., Ou, T., Key, C.C., Tong, S.H., Sequeira, R.C., Nelson, J.M., Nie, Y., Wang, Z., Boudyguina, E., et al. (2017). Dietary PUFAs attenuate NLRP3 inflammasome activation via enhancing macrophage autophagy. J Lipid Res 58, 1808-1821.

Sheth, S.S., Bodnar, J.S., Ghazalpour, A., Thipphavong, C.K., Tsutsumi, S., Tward, A.D., Demant, P., Kodama, T., Aburatani, H., and Lusis, A.J. (2006). Hepatocellular carcinoma in Txnip-deficient mice. Oncogene 25, 3528-3536.

Su, C., Shi, A., Cao, G., Tao, T., Chen, R., Hu, Z., Shen, Z., Tao, H., Cao, B., Hu, D., et al. (2015). Fenofibrate suppressed proliferation and migration of human neuroblastoma cells via oxidative stress dependent of TXNIP upregulation. Biochem Biophys Res Commun 460, 983988.

Szpigel, A., Hainault, I., Carlier, A., Venteclef, N., Batto, A.F., Hajduch, E., Bernard, C., Ktorza, A., Gautier, J.F., Ferre, P., et al. (2018). Lipid environment induces ER stress, TXNIP expression and inflammation in immune cells of individuals with type 2 diabetes. Diabetologia 61, 399-412.

Tomizawa, A., Hattori, Y., Inoue, T., Hattori, S., and Kasai, K. (2011). Fenofibrate suppresses microvascular inflammation and apoptosis through adenosine monophosphate-activated protein kinase activation. Metabolism 60, 513-522.

Villegas, V.E., and Zaphiropoulos, P.G. (2015). Neighboring gene regulation by antisense long non-coding RNAs. Int J Mol Sci 16, 3251-3266.

Wada, T., Gao, J., and Xie, W. (2009). PXR and CAR in energy metabolism. Trends Endocrinol Metab 20, 273-279. 
Waldhart, A.N., Dykstra, H., Peck, A.S., Boguslawski, E.A., Madaj, Z.B., Wen, J., Veldkamp, K., Hollowell, M., Zheng, B., Cantley, L.C., et al. (2017). Phosphorylation of TXNIP by AKT mediates acute influx of glucose in response to insulin. Cell Rep 19, 2005-2013.

Watanabe, R., Nakamura, H., Masutani, H., and Yodoi, J. (2010). Anti-oxidative, anti-cancer and anti-inflammatory actions by thioredoxin 1 and thioredoxin-binding protein-2. Pharmacol Ther $127,261-270$.

Wei, S., Fan, Q., Yang, L., Zhang, X., Ma, Y., Zong, Z., Hua, X., Su, D., Sun, H., Li, H., et al. (2017). Promotion of glycolysis by HOTAIR through GLUT1 upregulation via mTOR signaling. Oncol Rep 38, 1902-1908.

Wree, A., McGeough, M.D., Inzaugarat, M.E., Eguchi, A., Schuster, S., Johnson, C.D., Pena, C.A., Geisler, L.J., Papouchado, B.G., Hoffman, H.M., et al. (2017). NLRP3 inflammasome driven liver injury and fibrosis: Roles of IL-17 and TNF in mice. Hepatology 67, 736-749.

Wu, C.A., Huang, D.Y., and Lin, W.W. (2014). Beclin-1-independent autophagy positively regulates internal ribosomal entry site-dependent translation of hypoxia-inducible factor 1alpha under nutrient deprivation. Oncotarget 5, 7525-7539.

Wu, N., Zheng, B., Shaywitz, A., Dagon, Y., Tower, C., Bellinger, G., Shen, C.H., Wen, J., Asara, J., McGraw, T.E., et al. (2013). AMPK-dependent degradation of TXNIP upon energy stress leads to enhanced glucose uptake via GLUT1. Mol Cell 49, 1167-1175.

Yang, R., Wang, P., Chen, Z., Hu, W., Gong, Y., Zhang, W., and Huang, C. (2017). WY-14643, a selective agonist of peroxisome proliferator-activated receptor- $\alpha$, ameliorates lipopolysaccharide-induced depressive-like behaviors by preventing neuroinflammation and oxido-nitrosative stress in mice. Pharmacol Biochem Behav 153, 97-104.

Yoo, S.H., Abdelmegeed, M.A., and Song, B.J. (2013). Activation of PPAR $\alpha$ by Wy-14643 ameliorates systemic lipopolysaccharide-induced acute lung injury. Biochem Biophys Res Commun 436, 366-371.

Yoo, S.H., Park, O., Henderson, L.E., Abdelmegeed, M.A., Moon, K.H., and Song, B.J. (2011). Lack of PPAR $\alpha$ exacerbates lipopolysaccharide-induced liver toxicity through STAT1 inflammatory signaling and increased oxidative/nitrosative stress. Toxicol Lett 202, 23-29.

Yoshioka, J., Imahashi, K., Gabel, S.A., Chutkow, W.A., Burds, A.A., Gannon, J., Schulze, P.C., MacGillivray, C., London, R.E., Murphy, E., et al. (2007). Targeted deletion of thioredoxininteracting protein regulates cardiac dysfunction in response to pressure overload. Circ Res 101, 1328-1338.

Yuan, H., Xu, X., Feng, X., Zhu, E., Zhou, J., Wang, G., Tian, L., and Wang, B. (2019a). A novel long noncoding RNA PGC1beta-OT1 regulates adipocyte and osteoblast differentiation through antagonizing miR-148a-3p. Cell Death Differ. 
Yuan, Y., Cao, X.G., Hu, J., Li, J., Shen, D., You, L., Cui, X., Wang, X., Zhou, Y., Gao, Y., et al. (2019b). The role and possible mechanism of lncRNA AC092159.2 in modulating adipocyte differentiation. J Mol Endocrinol.

Zhang, C.L., Zhu, K.P., and Ma, X.L. (2017a). Antisense lncRNA FOXC2-AS1 promotes doxorubicin resistance in osteosarcoma by increasing the expression of FOXC2. Cancer Lett $396,66-75$.

Zhang, L., Ren, F., Zhang, X., Wang, X., Shi, H., Zhou, L., Zheng, S., Chen, Y., Chen, D., Li, L., et al. (2016). Peroxisome proliferator-activated receptor $\alpha$ acts as a mediator of endoplasmic reticulum stress-induced hepatocyte apoptosis in acute liver failure. Dis Model Mech 9, 799-809.

Zhang, Y., Foncea, R., Deis, J.A., Guo, H., Bernlohr, D.A., and Chen, X. (2014). Lipocalin 2 expression and secretion is highly regulated by metabolic stress, cytokines, and nutrients in adipocytes. PLoS One 9, e96997.

Zhang, Y., He, J., Zhao, J., Xu, M., Lou, D., Tso, P., Li, Z., and Li, X. (2017b). Effect of ApoA4 on SERPINA3 mediated by nuclear receptors NR4A1 and NR1D1 in hepatocytes. Biochem Biophys Res Commun 487, 327-332.

Zhao, G., Su, Z., Song, D., Mao, Y., and Mao, X. (2016). The long noncoding RNA MALAT1 regulates the lipopolysaccharide-induced inflammatory response through its interaction with NFkappaB. FEBS Lett 590, 2884-2895.

Zhao, Q., Yang, R., Wang, J., Hu, D.D., and Li, F. (2017). PPAR $\alpha$ activation protects against cholestatic liver injury. Sci Rep 7, 9967.

Zhao, X.Y., Xiong, X., Liu, T., Mi, L., Peng, X., Rui, C., Guo, L., Li, S., Li, X., and Lin, J.D. (2018). Long noncoding RNA licensing of obesity-linked hepatic lipogenesis and NAFLD pathogenesis. Nat Commun 9, 2986.

Zheng, S., Ren, X., Han, T., Chen, Y., Qiu, H., Liu, W., and Hu, Y. (2017). Fenofibrate attenuates fatty acid-induced islet $\beta$-cell dysfunction and apoptosis via inhibiting the NFkappaB/MIF dependent inflammatory pathway. Metabolism 77, 23-38.

Zhou, Z., Xu, M.J., and Gao, B. (2016). Hepatocytes: a key cell type for innate immunity. Cell Mol Immunol 13, 301-315. 


\section{Figure Legends}

Figure 1. LncRNA identified as PPARA targets are expressed in several oxidative tissues but exhibit liver-specific transcriptional response to WY-14643

(A) Venn diagrams of RefSeq transcripts and lncRNA transcripts that were differentially regulated by WY-14643 (fold change $>2$ at FDR < 0.05) in wild-type and in Ppara ${ }^{-/-}$mouse liver, as determined by RNA-seq. 123 of the RefSeq genes are non-coding, indicated by their NR accession numbers. Six RefSeq genes and six lncRNA genes show opposite responses to WY-14643 treatment and are excluded from the gene counts shown. (B) Relative basal lncRNA expression in select tissues. (C) LncRNA expression in tissues from Ppara $^{+/+}$and Ppara $^{-/-}$mice treated with WY-14643 for 48 hours. At least five mice were analyzed for each genotype and treatment group. Each data point represents the mean $\pm \mathrm{SD}$ for $\mathrm{n}=5$ tissue samples. $\# P<0.05$. Abbreviation: ND, Not Detected.

Figure 2. Txnip and IncRNA Gm15441 are inversely regulated following PPARA activation. Wild-type $\left(\mathrm{Ppara}^{+/+}\right)$mice were treated with WY-14643 and stranded RNA-seq was performed on total liver mRNA. (A) Expression of the antisense lncRNA Gm15441 is strongly upregulated. (B) Expression of the protein-coding Txnip mRNA is downregulated. Shown are the changes in expression of Gm15441 (C) and Txnip (D) mRNAs in Ppara ${ }^{+/+}$and Ppara $^{-/-}$mice treated with WY14643, or vehicle control, for 48 hours, determined by qRT-PCR. (E) Time course for changes in expression of Gm15441 and Txnip mRNA over a 24 hours period following treatment with WY14643 by gavage, determined by qRT-PCR. The maximum response of Txnip mRNA was seen at 1.5 hours and for Gm15441 was seen at 6 hours. Each data point represents the mean \pm SD for $\mathrm{n}=$ 5 liver samples. $* \mathrm{P}<0.05 ; * * \mathrm{P}<0.01 ; * * * \mathrm{P}<0.001$.

\section{Figure 3. LncRNA Gm15441 is a direct PPARA target gene.}

(A) PPARA ChIP-seq read peaks from agonist (GW7647)-treated mouse liver. (B) Schematic representation of seven PPRE sequences found within the Gm 15441 promoter $(-10 \mathrm{~kb})$. ChIP primer binding sites and reporter gene construct inserts are shown. (C) Luciferase-based reporter assays identified five functional PPREs within the Gm15441 promoter, based on $\mathrm{n}=3$ replicates. (D) PPARA ChIP assays assessed PPRE binding in liver samples from Ppara $^{+/+}$and Ppara $^{-/-}$mice treated with WY-14643. Experiments were performed with at least four different livers. Rabbit IgG and antibody to histone protein $\mathrm{H} 3$ were used as negative and positive controls, respectively. 
Each data point represents the mean $\pm \mathrm{SD}$ for $\mathrm{n}=5$ liver samples. $* \mathrm{P}<0.05 ; * * \mathrm{P}<0.01 ; * * * \mathrm{P}<$ 0.001 for comparisons to pGL4.11 empty vector (C) or as indicated (D). Abbreviation: ns, not significant.

\section{Figure 4. Generation of the Gm15441-null mouse line.}

Targeting strategy for generating a strand-specific Gm15441-null mouse line. (A) Exon structure of targeted Gm15441-Txnip locus. (B) CRISPR/Cas9-mediated insertion of Lox-STOP-Lox (LSL) cassette selectively ablates Gm15441 expression (C) Cre-mediated removal of STOP cassette rescues Gm15441 expression. (D) Gm15441 knockout mouse genotyping. (E) Analysis of Gm15441 mRNA by qRT-PCR from livers of $G m 15441^{+/+}, G m 15441^{+/}$, and $G m 15441^{\mathrm{LSL}}$ mice. Each data point represents the mean $\pm \mathrm{SD}$ for $\mathrm{n}=5$ liver samples. ${ }^{*} * \mathrm{P}<0.01$; $* * * \mathrm{P}<0.001$. (F) Fluorescence in situ hybridization staining of Gm15441 RNA in livers of $\mathrm{Gm}_{15441^{+/+}}$and Gm15441 ${ }^{\text {LSL }}$ mice treated with WY-14643 for 48 hours. Scale bars represents $20 \mathrm{~nm}$ (200x).

\section{Figure 5. Loss of $G m 15441$ potentiates inflammasome activation by WY-14643-induced metabolic stimulation.}

(A) Physiological endpoints from $G m 15441^{+/+}$and $G m 15441^{\text {LSL }}$ mice treated with WY-14643 for 48 hours. Liver indexes (mg liver/g body mass) in response to WY-14643 treatment. (B) H\&E staining of liver tissue. Scale bars represents $100 \mathrm{~nm}$ (400x). (C) Analysis of Acadm, Cyp4a14, Txnip, and Gm15441 mRNAs in livers of $G m 15441^{+/+}$and $G m 15441^{\mathrm{LSL}}$ mice treated with WY14643 for 48 hours, measured by qRT-PCR. (D) Analysis of TXNIP, TUBA1B, CASP1 (cleaved), and IL1B (cleaved) protein on livers from $G m 15441^{+/+}$and $G m 15441^{\text {LSL }}$ mice treated with WY14643 for 48 hours. (E) Densitometric analysis of TXNIP, CASP1, and IL1B protein levels. Each data point represents mean $\pm \mathrm{SD}$ for $\mathrm{n}=5$ liver samples. $* \mathrm{P}<0.05 ; * * \mathrm{P}<0.01 ; * * * \mathrm{P}<0.001$; $* * * * \mathrm{P}<0.0001$ for comparisons between WY-14643-stimulated and unstimulated livers of the same genotype, or as shown (dashed horizontal lines).

Figure 6. Loss of $G m 15441$ potentiates inflammasome activation during physiological response to acute fasting.

(A) Physiological endpoints from $G m 15441^{+/+}$and $G m 15441^{\mathrm{LSL}}$ mice after 24 hours fasting. Liver indexes (mg liver/g body mass) in response to fasting. (B) ORO staining of liver tissues after a 24 
$\mathrm{h}$ fast. Scale bars represents $100 \mathrm{~nm}(200 \mathrm{x})$. (C) TG and CHOL levels from serum and liver tissues after a 24 h fast. (D) Analysis of Apoa4, Bhmt, Lcn2, Orm2, Saa1, and Saa 2 mRNAs in livers of $G m 15441^{+/+}$and $G m 15441^{\mathrm{LSL}}$ mice after $24 \mathrm{~h}$ fast. Each data point represents the mean \pm SD for $\mathrm{n}=5$ liver samples. $* \mathrm{P}<0.05 ; * * \mathrm{P}<0.01 ; * * * \mathrm{P}<0.001$ for comparisons between fast-stimulated and unstimulated livers of the same genotype, or as shown (dashed horizontal lines).

\section{Figure 7. Gm15441 regulates TXNIP translation in part through IRES sequences found within the 5' UTR of TXNIP.}

(A) Schematic construct of inserts for TXNIP of non-5'UTR and 5'UTR sequence containing GFP. (B) Analysis of Gm15441 and Gfp mRNA in Hepa-1 cells. (C) Analysis of GFP protein and relative density of GFP in Hepa-1 cells (right). (D and E) Fluorescence of GFP in Hepa-1 cells transfected with EGFP or 5' UTR sequence containing EGFP with empty or Gm15441 plasmid DNA for 48 hours. Scale bars represents $20 \mathrm{~nm}$ (100x). (F) Analysis of Hfe2, Pol3gl and Ankrd34a mRNAs from Hepa-1 and NIHT3T cells transfected with empty or Gm15441 expression vector for 24 hours. Each data point represents mean $\pm \mathrm{SD}$ for $\mathrm{n}=3$ replicates. $* \mathrm{P}<0.05 ; * * * \mathrm{P}<0.001$ for comparisons in the absence of $\operatorname{Gm} 15441(\mathrm{~B}, \mathrm{C})$ or to empty vector $(\mathrm{F})$. (G) Model for role of GM15441 in suppressing TXNIP-mediated inflammasome activation.

\section{Supplemental Legends}

Table S1A. The list of differential gene expression analysis of RefSeq genes. (Related to Figure 1)

Table S1B. The list of differential gene expression analysis of IncRNA genes. (Related to Figure 1)

Table S1C. LncRNAs responsive to activator of PPARA, CAR and PXR in mouse liver. (Related to Figure 1) Groups \#1, \#2: lncRNAs that are consistently induced, or consistently repressed, by all 3 nuclear receptors, as indicated; Groups \#3, \#4: lncRNAs consistently induced or repressed by PPARA and by CAR, but not by PXR; Groups \#5, \#6: lncRNAs consistently induced or repressed by PPARA and by PXR, but not by CAR; Groups \#7, \#8: lncRNAs induced or repressed by PPARA but showing the opposite responses to activators of CAR, PXR, or both receptors. 
Table S1D. Pathway analysis of upregulated genes from the WY-14643 treated mice liver. (Related to Figure 1)

Table S1E. Pathway analysis of downregulated genes from the WY-14643 treated mice liver. (Related to Figure 1)

Table S2. The list of differential gene expression analysis of RefSeq genes from Gm15441+/+ (WT) and Gm15441LSL (KO) mouse liver. (Related to Figure 6)

Table S3. Gm15441 donor plasmid sequence. (Related to Figure 4)

Table S4. List of primer sequences used in qPCR and cloning

Table S5. Gm15441 target sequence used for fluorescence in situ hybridization probes. (RED: Excluded sequence that is antisense to Txnip) (Related to Figure 4)

Figure S1. PPARA-dependent IncRNA expression profiles are analogous to protein coding genes. (Related to figure 1) Ppara $^{+/+}$mice were gavaged with the PPARA agonist WY-14643 (50 $\mathrm{mg} / \mathrm{kg}$ ). Livers were collected at $\mathrm{t}=0,1.5,3,6,12$, and 24 hours. (A) qRT-PCR analysis of upregulated protein cording PPARA target genes response by WY-14643 treatment timedependently. (B) qRT-PCR analysis of down-regulated protein coding gene response by WY14643 treatment time-dependently. (C) qRT-PCR analysis of up-regulated PPARA target lncRNAs response by WY-14643 treatment time-dependently. (D) qRT-PCR analysis of downregulated PPARA target lncRNAs response by WY-14643 treatment time-dependently.

Figure S2. Loss of Gm15441 potentiates inflammasome activation during physiological response to acute fasting. (Related to figure 6) (A) Analysis of Acadm, Cyp4a14, and Txnip mRNA and Gm15441 expression from livers of fasted $G m 15441^{+/+}$and $G m 15441^{\mathrm{LSL}}$ mice, determined by qRT-PCR. (B) Analysis of TXNIP, CASP1 and IL1B protein expression from livers of fasted $G m 15441^{+/+}$and $G m 15441^{\mathrm{LSL}}$ mice. (C) Densitometric analysis of TXNIP, CASP1 and IL1B protein expression. (D) H\&E staining of liver tissues after $24 \mathrm{~h}$ fast. Scale bars represents $100 \mathrm{~nm}(200 \mathrm{x})$. Each data point represents the mean $\pm \mathrm{SD}$. $* \mathrm{P}<0.05 ; * * \mathrm{P}<0.01 ; * * * \mathrm{P}<0.001$. 
Graphical abstract

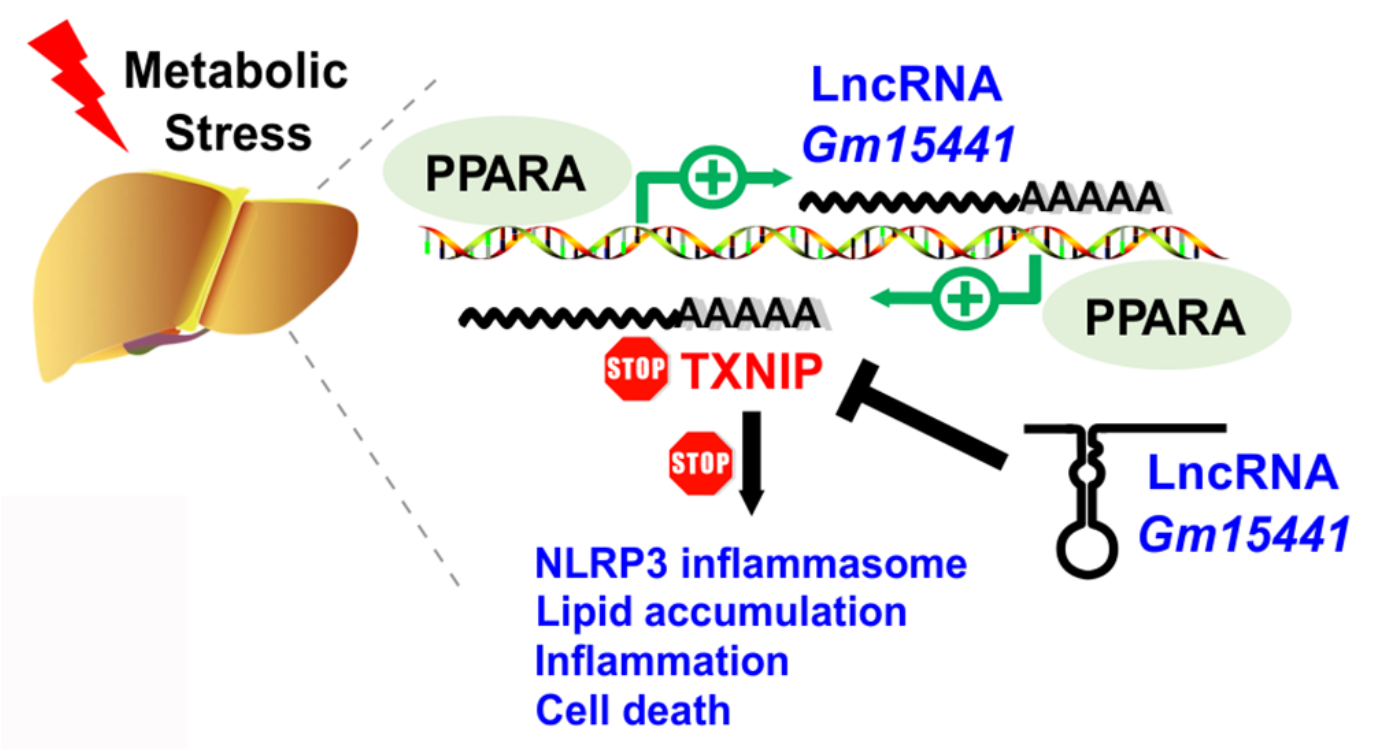

\section{Highlights}

PPARA regulates expression of more than 400 liver-expressed long coding RNAs

Gm15441 is PPARA-dependent lncRNA that is antisense transcript to TXNIP

Gm15441 expression suppresses TXNIP-mediated NLRP3 inflammasome activation

Gm15441 attenuates hepatic inflammation against metabolic stress

\section{In brief}

Fasting is known to elicit anti-inflammatory effects. The molecular mechanisms that thwart inflammation during caloric restriction are not well understood and may represent promising new therapeutic targets. Herein, Brocker, Kim and colleagues identify a novel regulatory loop comprised a long non-coding RNA that suppresses expression of a proinflammatory protein.

\section{Supplemental Information Titles and Legends}

Supplemental Information includes 2 figures and 5 tables and can be found with this article online at XXX 


\section{Figure 1}

A Up regulated Down regulated

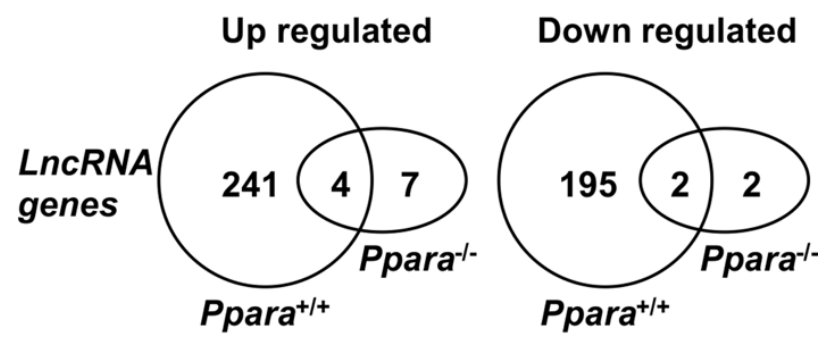

B
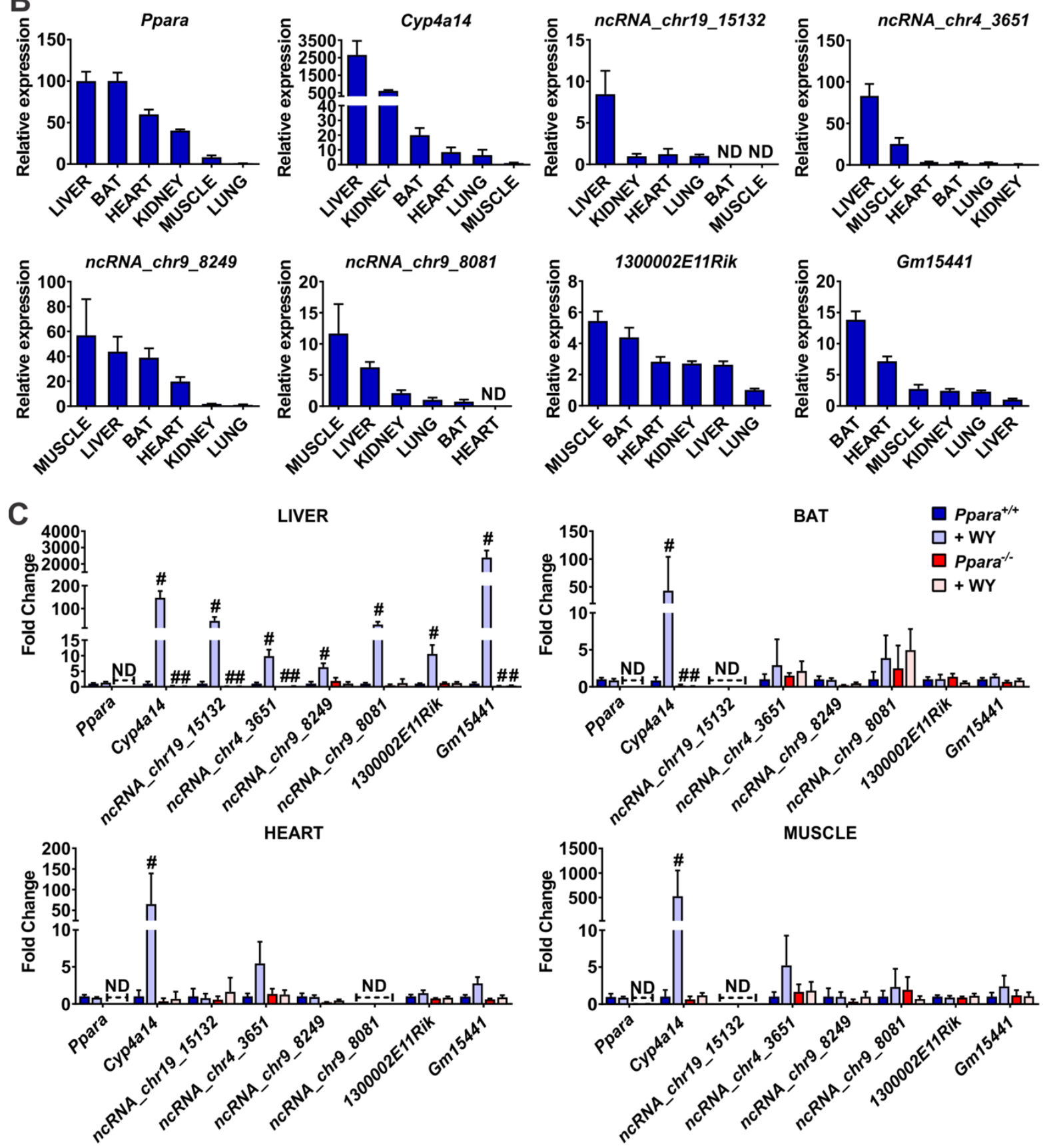
bioRxiv preprint doi: https://doi org/101101/675785; this version posted June 20, 2019. The copyright holder for this preprint (which was not certified by peer review) is the author/funder, who has granted bioRxiv a license to display the preprint in perpetuity. It is made available under aCC-BY-NC-ND 4.0 International license.

\section{Figure 2}

A ReVerse StRand (-) Gm15441 upregulation

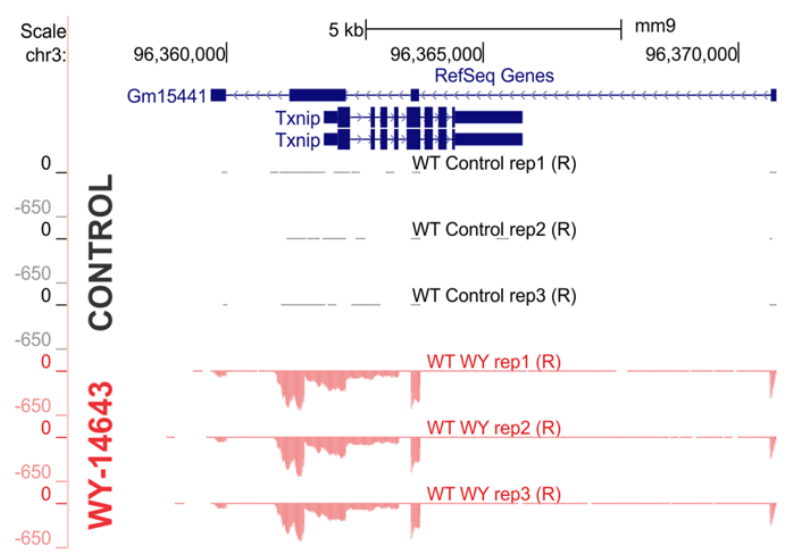

B FORWARD STRAND (+) Txnip suppression

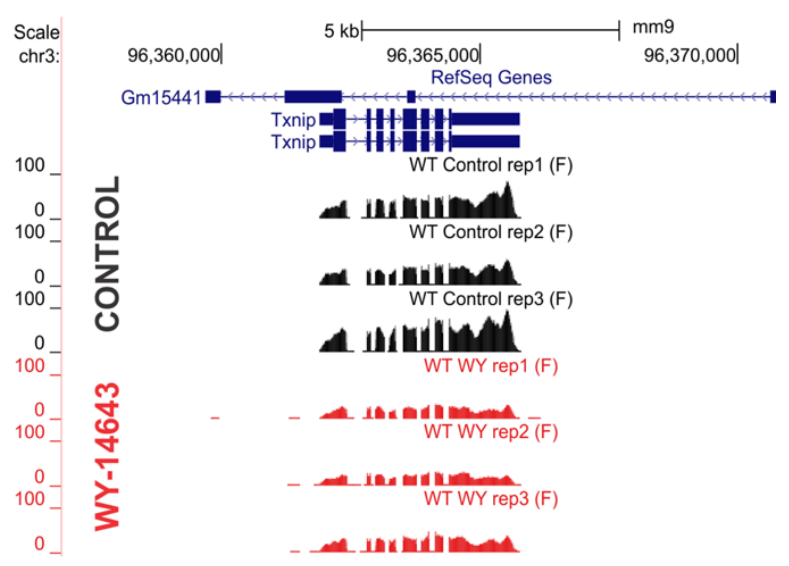

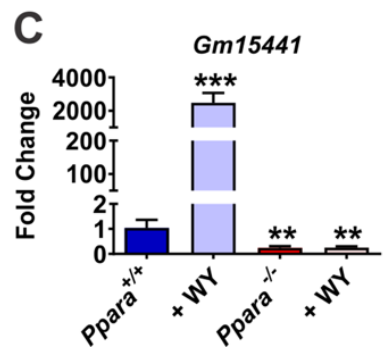
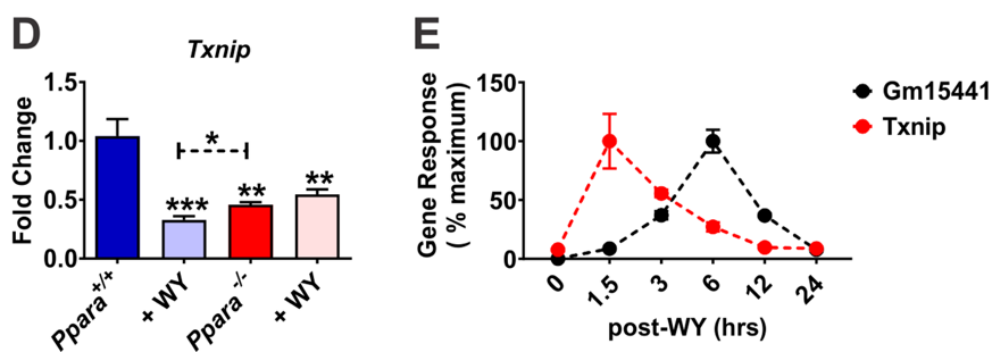


\section{Figure 3}

A Gm15441 promoter mapped PPARA ChIP-seq reads

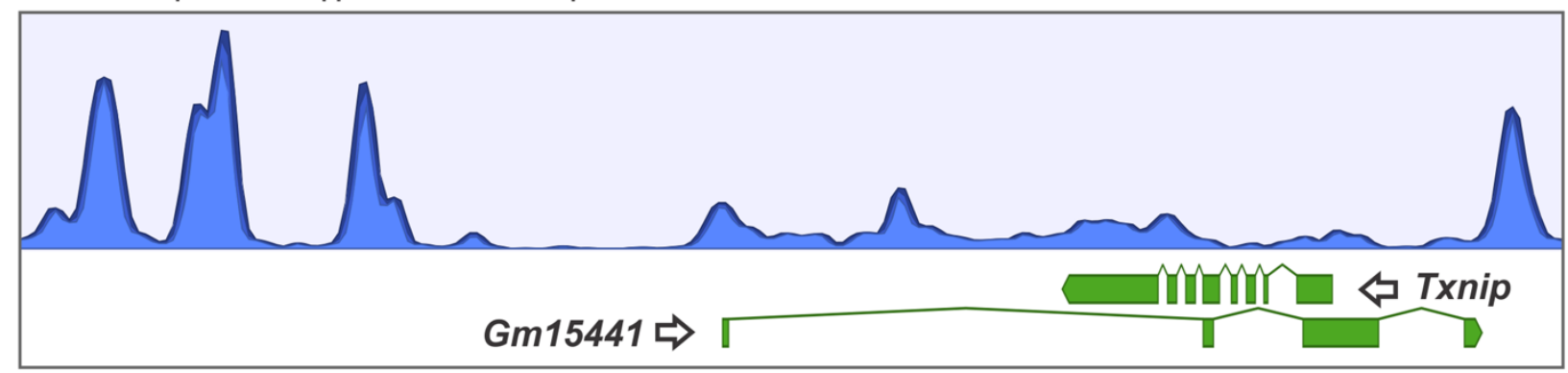

B Predicted PPREs in Gm15441 promoter (NR_040409)
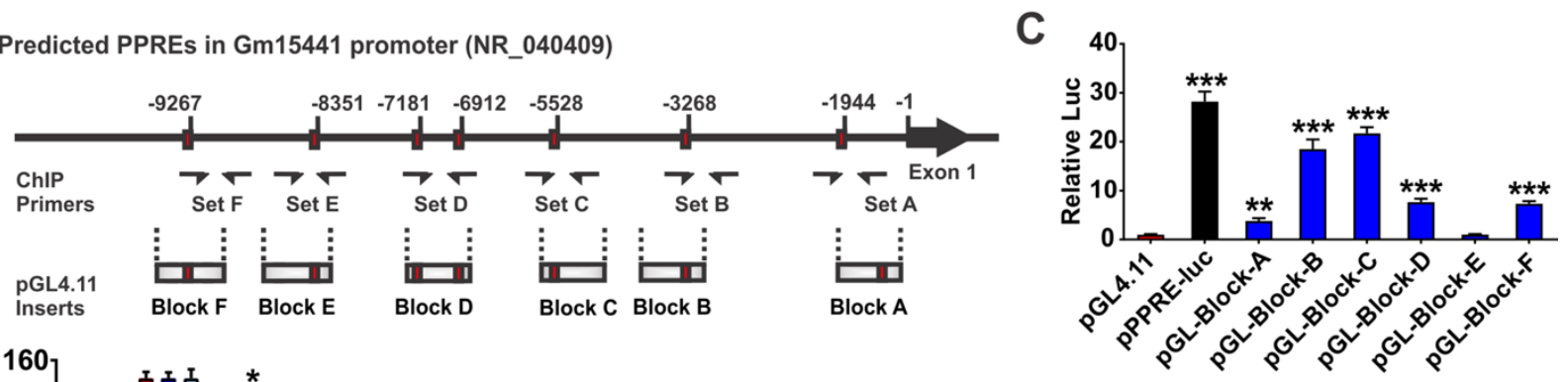

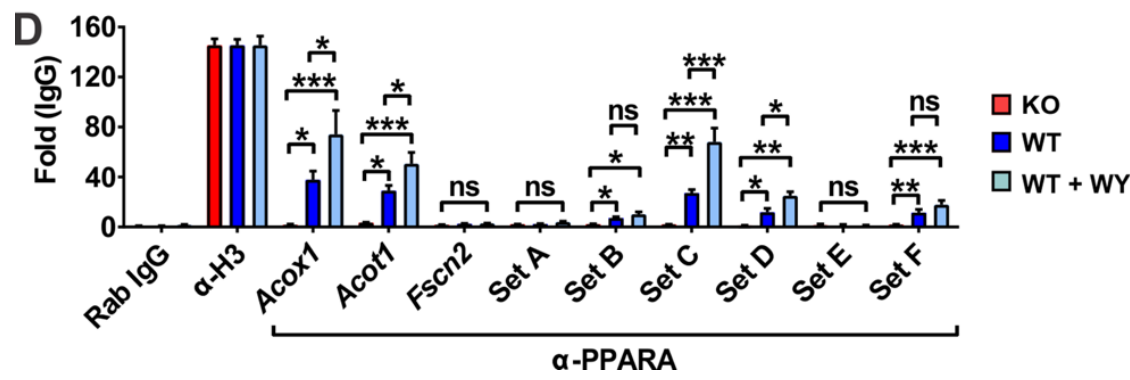


bioRxiv preprint doi: https://doi.org/10.1101/675785; this version posted June 20, 2019. The copyright holder for this preprint (which was not certified by peer review) is the author/funder, who has granted bioRxiv a license to display the preprint in perpetuity. It is made available under aCC-BY-NC-ND 4.0 International license.

\section{Figure 4}

AGm15441-Txnip Locus

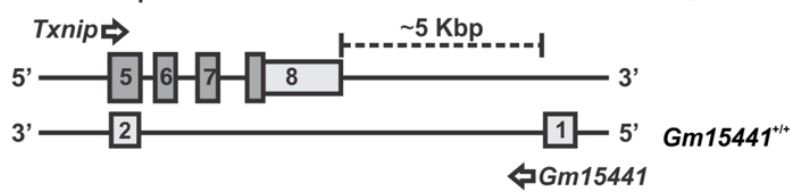

CRISPR/Cas9-mediated Lox-STOP-Lox (LSL) Insert

B OFF Allele (Gm15441 ${ }^{\text {LLL }}$ )

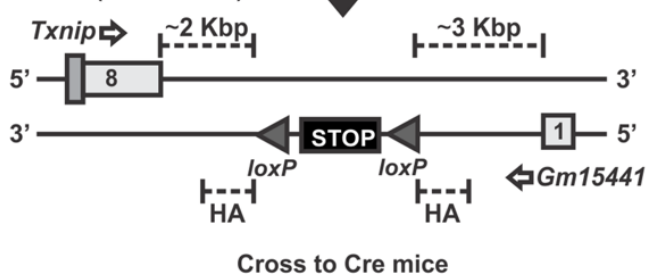

Con Allele (Gm15441 ${ }^{\text {Lox }}$ )

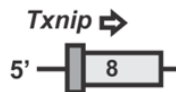

$$
\text { 3' }
$$

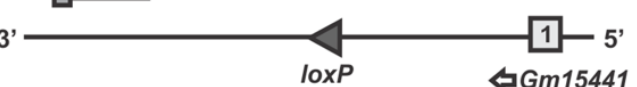

D Genotyping

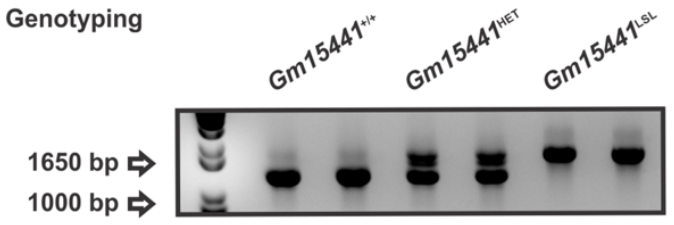

\section{E}

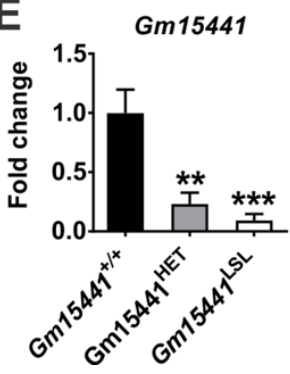

\section{F}

Gm15441

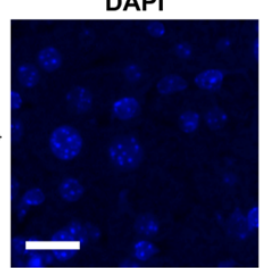

$$
\text { . }
$$
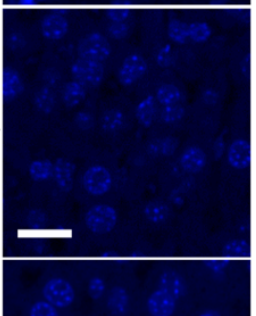

Gm15441 + WY

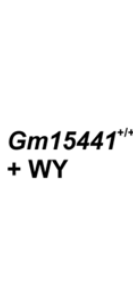

Gm15441 $1^{\text {sL }}$ +WY
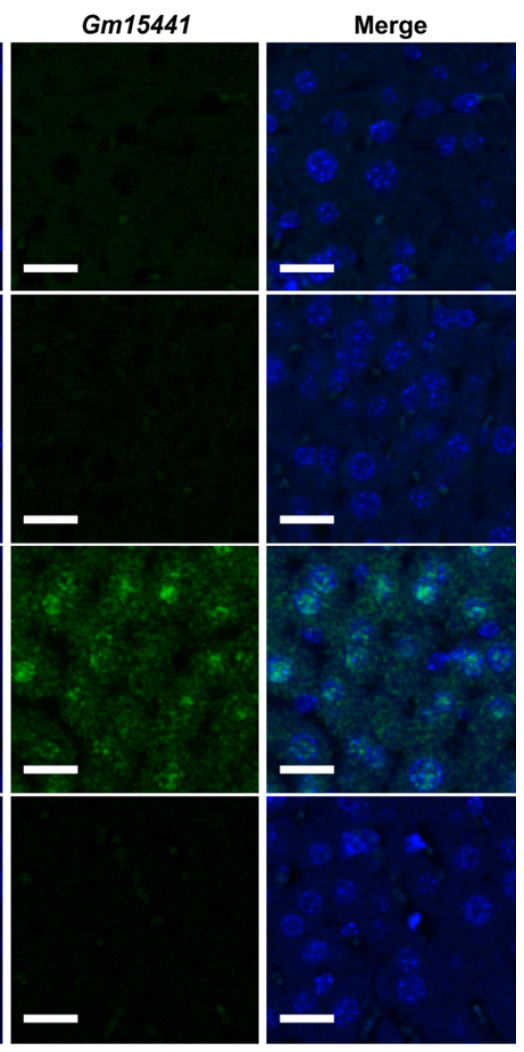


\section{Figure 5}

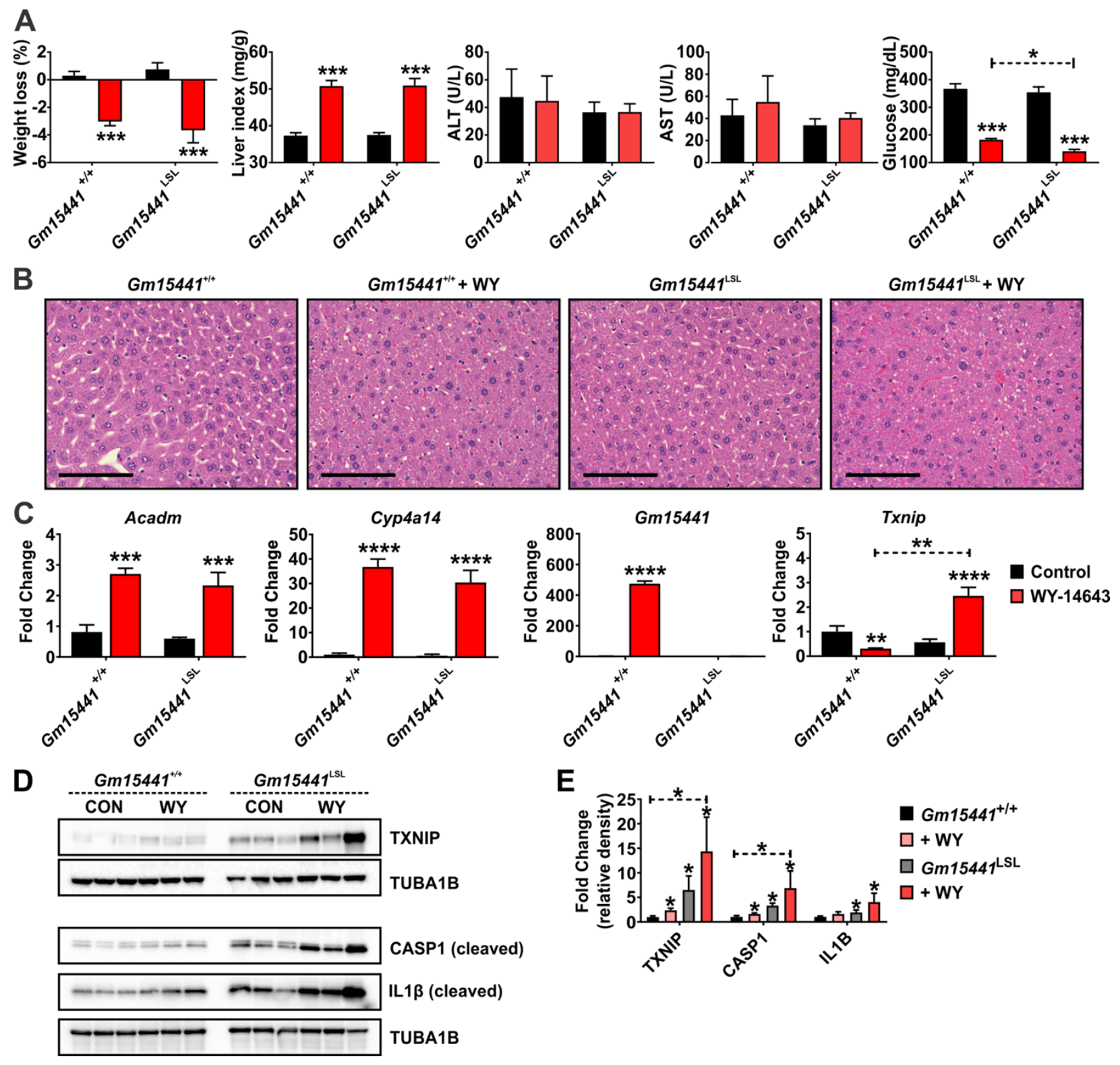




\section{Figure 6}

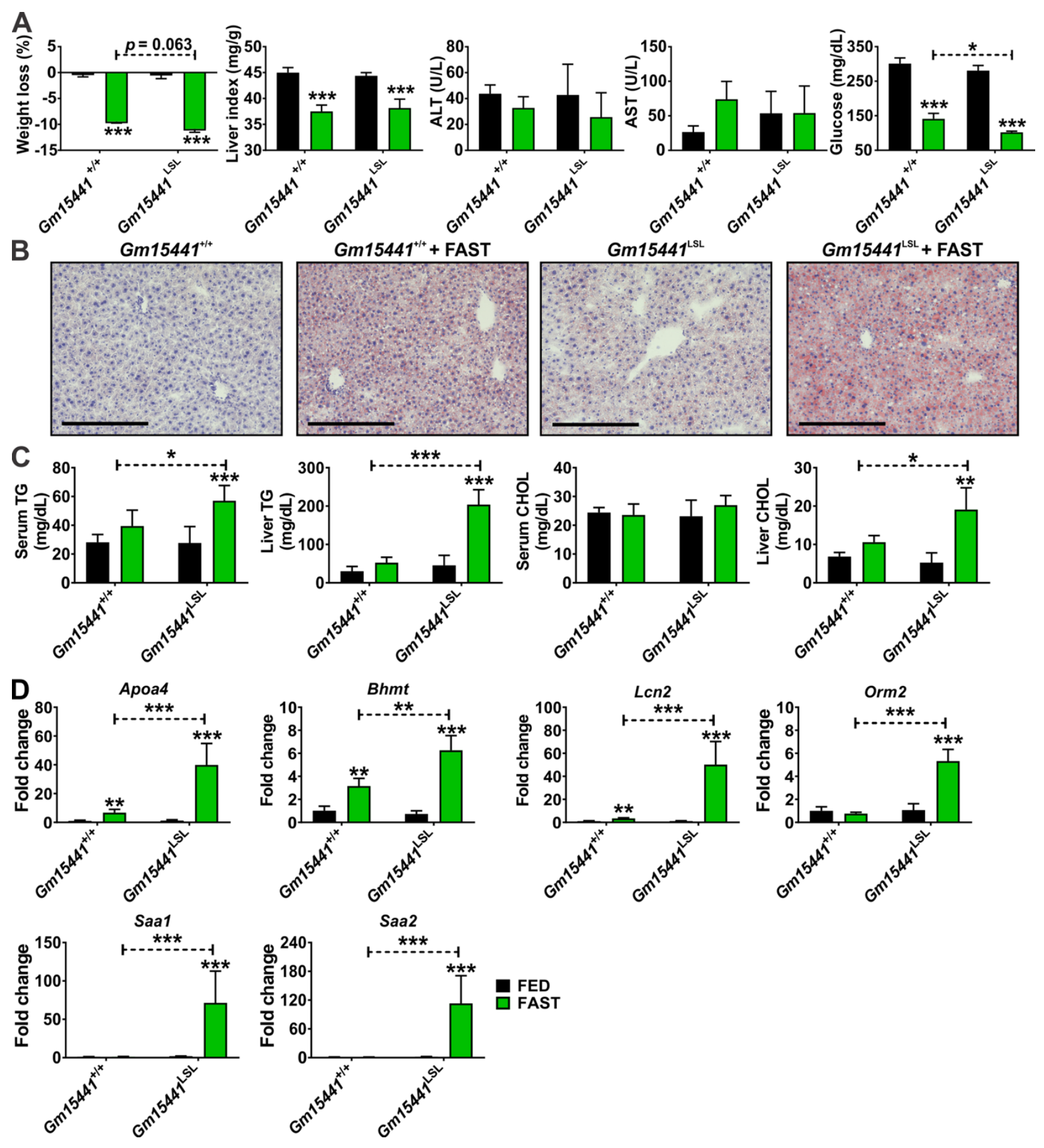




\section{Figure 7}

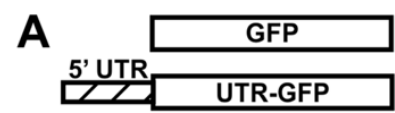

B

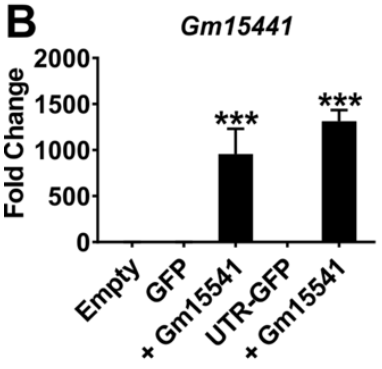

C
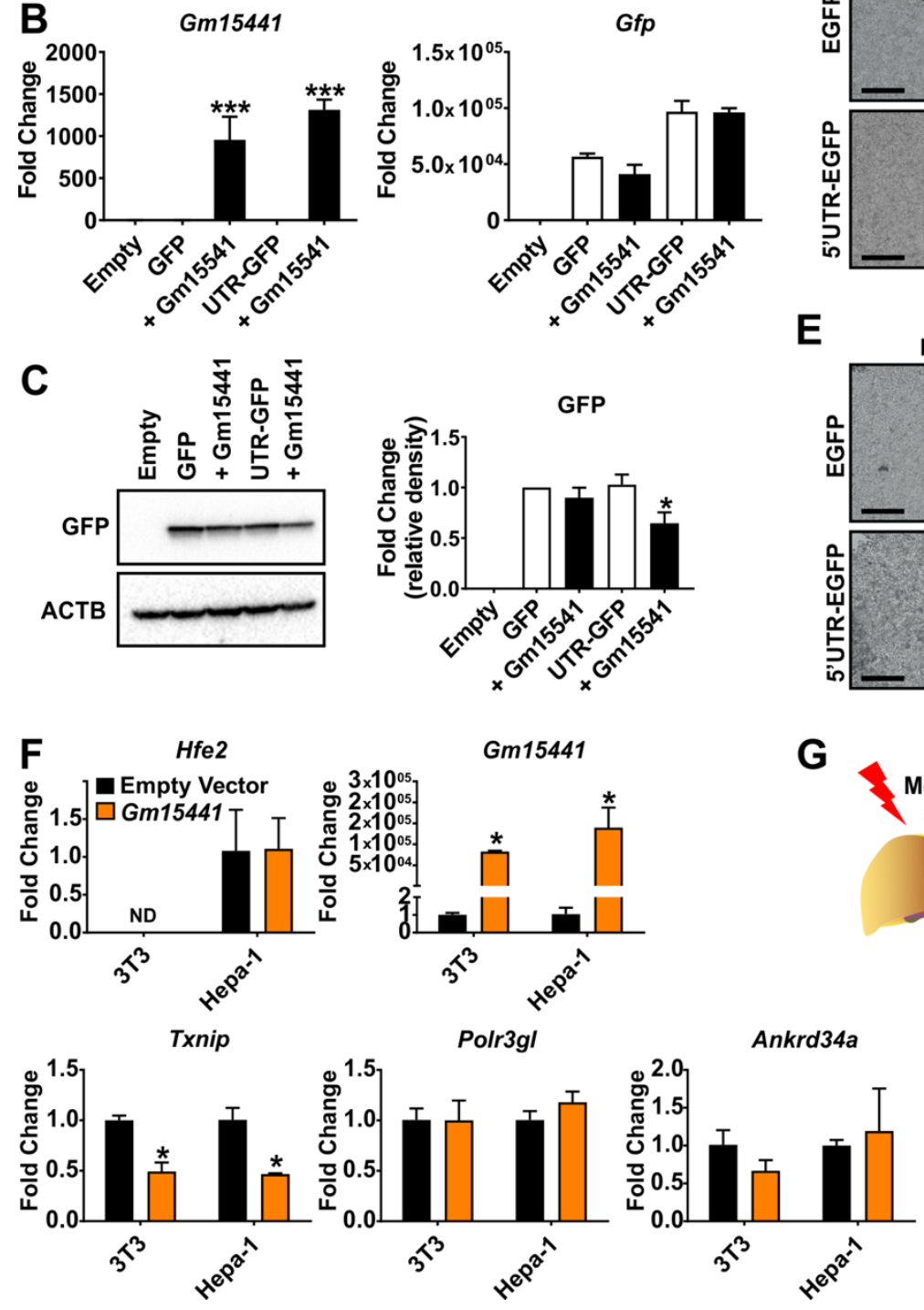
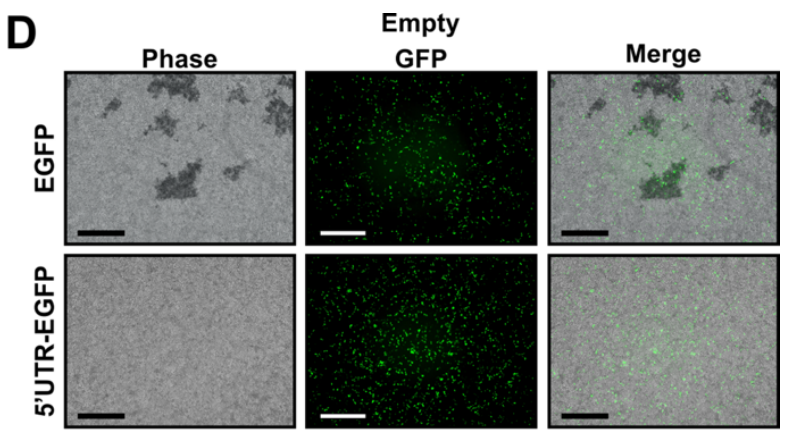

E
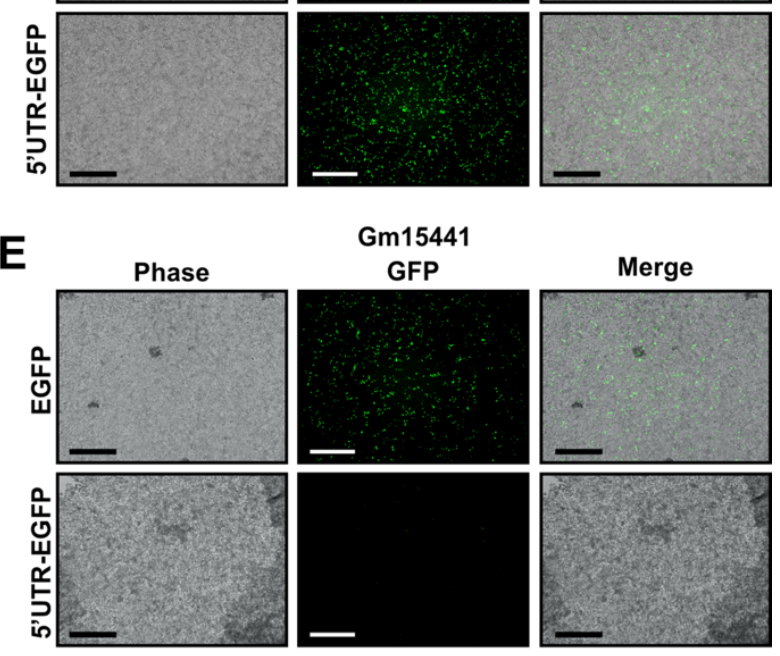

G
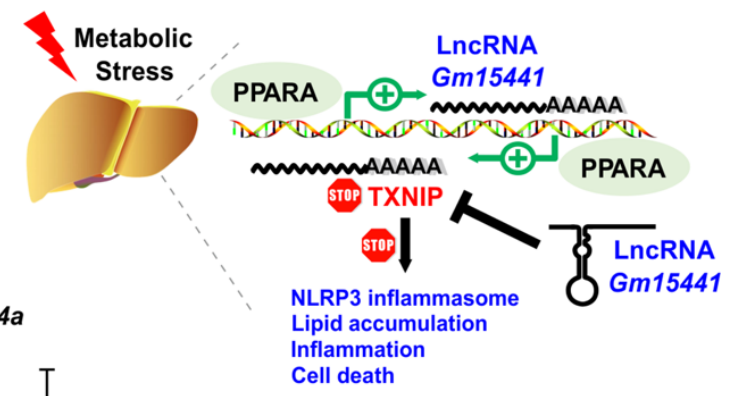


\section{Supplemental Figures and Tables}

A Protein-coding (peak@6 hrs)

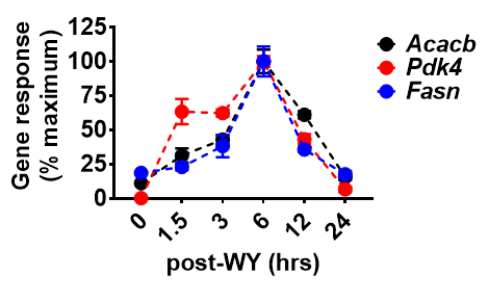

B Protein-coding (decline @ 3-6 hrs)

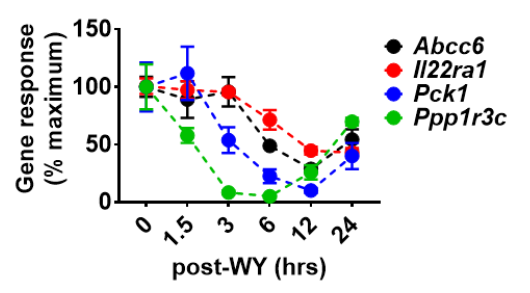

C IncRNA (peak @ 6 hrs)

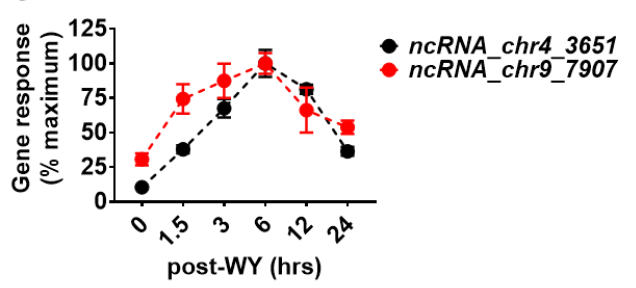

D IncRNA (decline @ 3-6 hrs)

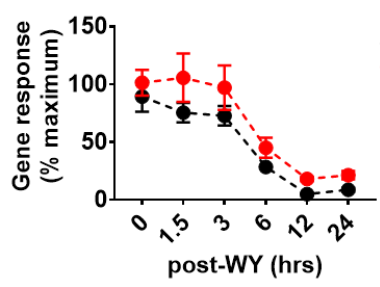

Protein-coding (peak @ 6-12 hrs)

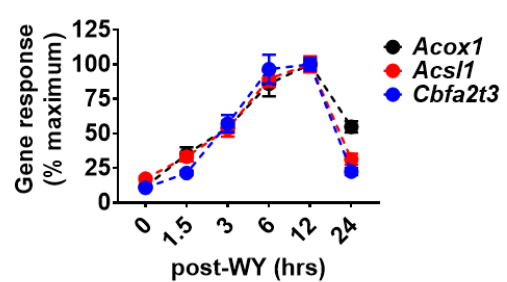

Protein-coding (decline @ 12 hrs)

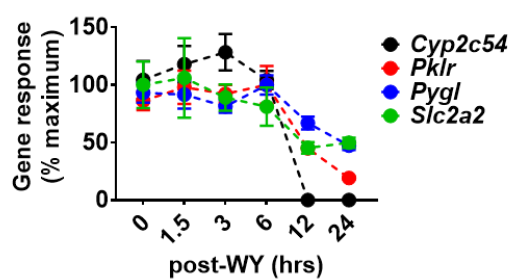

IncRNA (peak @ 6-12 hrs)

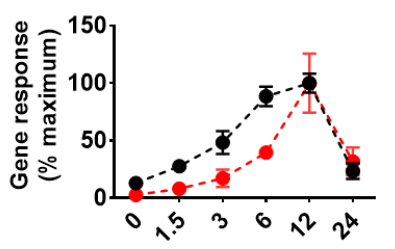

IncRNA (decline @ 12 hrs)

- ncRNA_chr16 13170
ncRNA_chr8_7528

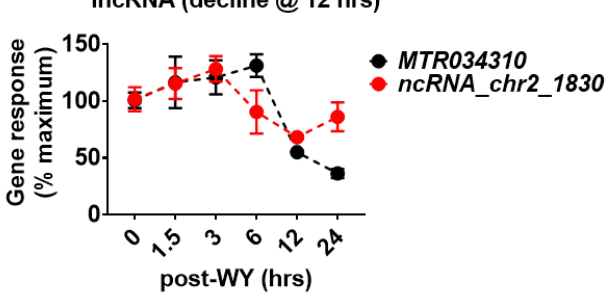

Protein-coding (peak @ 12 hrs)

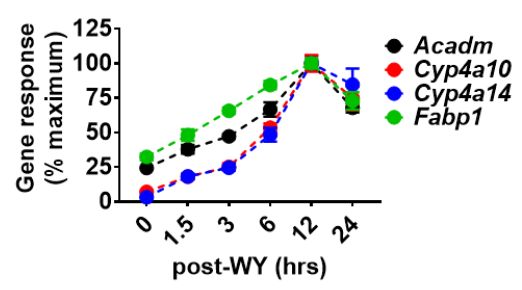

Protein-coding (decline @ 24 hrs)

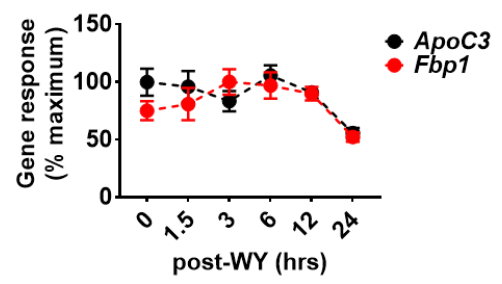

E11Rik

- ncRNA_chr9_8081

Figure S1. PPARA-dependent IncRNA expression profiles are analogous to protein coding

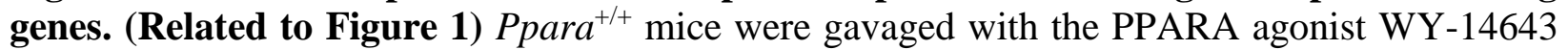
$(50 \mathrm{mg} / \mathrm{kg})$. Livers were collected at $\mathrm{t}=0,1.5,3,6,12$, and 24 hours. (A) qRT-PCR analysis of up-regulated protein cording PPARA target genes response by WY-14643 treatment timedependently. (B) qRT-PCR analysis of down-regulated protein coding gene response by WY14643 treatment time-dependently. (C) qRT-PCR analysis of up-regulated PPARA target IncRNAs response by WY-14643 treatment time-dependently. (D) qRT-PCR analysis of downregulated PPARA target IncRNAs response by WY-14643 treatment time-dependently. 

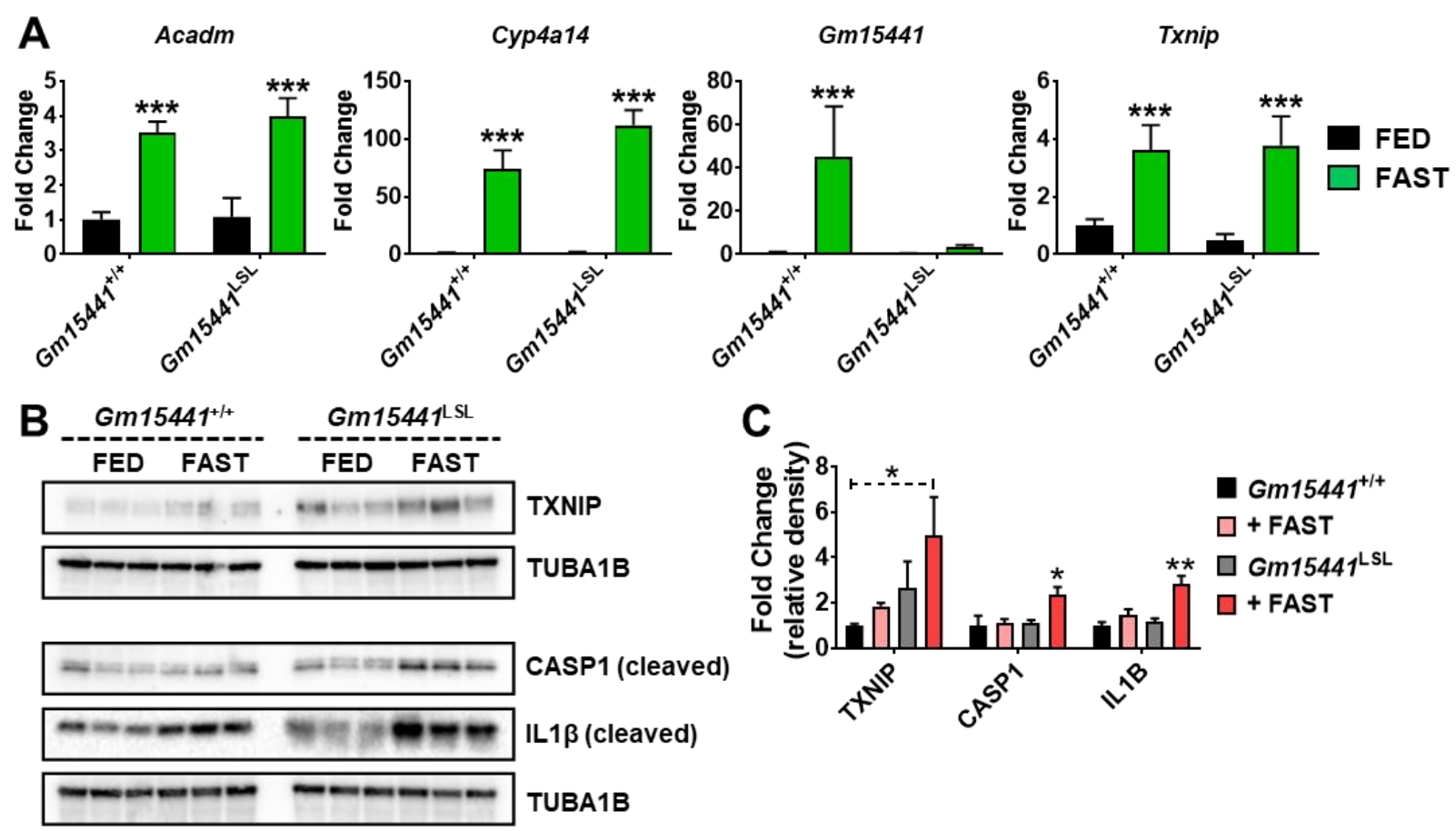

C
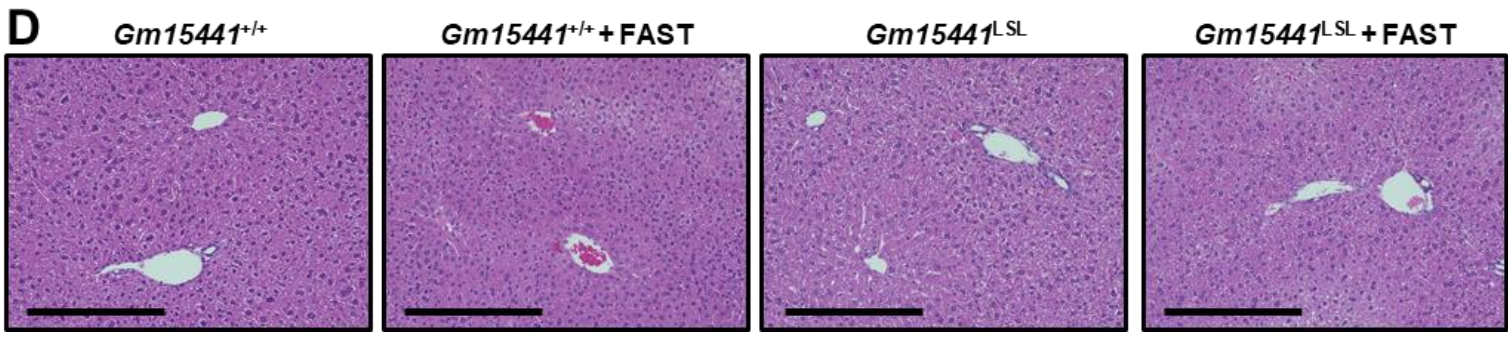

Figure S2. Loss of Gm15441 potentiates inflammasome activation during physiological response to acute fasting. (Related to Figure 6) (A) Analysis of Acadm, Cyp4a14, and Txnip mRNA and Gm15441 expression from livers of fasted $G m 15441^{+/+}$and $G m 15441^{\mathrm{LSL}}$ mice, determined by qRT-PCR. (B) Analysis of TXNIP, CASP1, and IL1B protein expression from livers of fasted $\mathrm{Gm} 15441^{+/+}$and $\mathrm{Gm} 15441^{\mathrm{LSL}}$ mice. (C) Densitometric analysis of TXNIP, CASP1, and IL1B protein expression. (D) H\&E staining of liver tissues after $24 \mathrm{~h}$ fast. Scale bars represents $100 \mathrm{~nm}(200 \mathrm{x})$. Each data point represents the mean $\pm \mathrm{SD}$. *P $<0.05 ; * * \mathrm{P}<0.01 ; * * * \mathrm{P}<0.001$. 